ARTICLE

\title{
2-Oxoglutarate derivatives can selectively enhance or inhibit the activity of human oxygenases
}

Yu Nakashima 1,2,3, Lennart Brewitz (1) 1,3, Anthony Tumber ${ }^{1}$, Eidarus Salah ${ }^{1} \&$ Christopher J. Schofield (D) ${ }^{1 凶}$

2-Oxoglutarate (2OG) oxygenases are validated agrochemical and human drug targets. The potential for modulating their activity with 20G derivatives has not been explored, possibly due to concerns regarding selectivity. We report proof-of-principle studies demonstrating selective enhancement or inhibition of $20 \mathrm{G}$ oxygenase activity by 2-oxo acids. The human $20 \mathrm{G}$ oxygenases studied, factor inhibiting hypoxia-inducible transcription factor $\mathrm{HIF}-\alpha(\mathrm{FIH})$ and aspartate/asparagine- $\beta$-hydroxylase $(\mathrm{AspH})$, catalyze $\mathrm{C} 3$ hydroxylations of Asp/Asnresidues. Of 35 tested $20 \mathrm{OG}$ derivatives, 10 enhance and 17 inhibit FIH activity. Comparison with results for $\mathrm{AspH}$ reveals that $2 \mathrm{OG}$ derivatives selectively enhance or inhibit $\mathrm{FIH}$ or $\mathrm{AspH}$. Comparison of $\mathrm{FIH}$ structures complexed with $2 \mathrm{OG}$ derivatives to those for $\mathrm{AspH}$ provides insight into the basis of the observed selectivity. 2-Oxo acid derivatives have potential as drugs, for use in biomimetic catalysis, and in functional studies. The results suggest that the in vivo activity of $20 \mathrm{O}$ oxygenases may be regulated by natural 2-oxo acids other than $20 \mathrm{OG}$.

\footnotetext{
${ }^{1}$ Chemistry Research Laboratory, Department of Chemistry and the Ineos Oxford Institute for Antimicrobial Research, University of Oxford, 12 Mansfield Road, OX1 3TA Oxford, UK. ${ }^{2}$ Present address: Institute of Natural Medicine, University of Toyama, 2630-Sugitani, 930-0194 Toyama, Japan. ${ }^{3}$ These authors contributed equally: Yu Nakashima, Lennart Brewitz. ${ }^{{ }_{\text {email: }}}$ christopher.schofield@chem.ox.ac.uk
} 


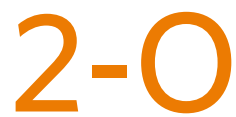
xoglutarate (2OG) and Fe(II)-dependent oxygenases couple substrate oxidation with $2 \mathrm{OG}$ decarboxylation to give succinate and $\mathrm{CO}_{2}$; they have important functions in human biology, e.g., in hypoxia signaling, extracellular matrix biosynthesis, lipid metabolism, and DNA/RNA damage repair ${ }^{1}$. Nature appears to have evolved complex mechanisms to regulate the activity of, at least, some of the human 2OG oxygenases, including sometimes by the use of small-molecules ${ }^{2-6}$. In cancer cells, normal regulation of $2 \mathrm{OG}$ oxygenase activity can be dysregulated, inter alia owing to elevated levels of tricarboxylic acid (TCA) cycle and related metabolites, such as $(R)$-2-hydroxyglutarate $(2 \mathrm{HG})^{6-8}$. The presence of 2HG has been correlated with ten-eleven-translocation (TET) and JmjC lysine-specific $N^{\varepsilon}$-demethylases (JmjC KDMs) inhibition; in vitro studies revealed that $2 \mathrm{HG}$ inhibits these $2 \mathrm{OG}$ oxygenases in a 2OG-competitive manner ${ }^{4,9}$. By contrast with the extensive studies on 2HG- and TCA cycle intermediate-mediated inhibition of 2OG oxygenases, only one human metabolite other than $2 \mathrm{OG}$ itself, i.e., 2 -oxoadipate (2OA), has been reported to enable catalysis by wild-type $2 \mathrm{OG}$ oxygenases by acting as a cosubstrate ${ }^{10-13}$.

Despite the importance of 2-oxo acids in biology, medicine, and synthetic chemistry ${ }^{14-18}$, few studies have been directed at identifying 2-oxo acids other than $2 \mathrm{OA}$ which retain the catalytic activity of human 2OG oxygenases. C4-alkyl-substituted 2OG derivatives have been investigated as alternative cosubstrates for human JmjC lysine-specific $N^{\varepsilon}$-demethylase $4 \mathrm{~A}$ (KDM4A), however, activity was observed with active site mutated, but not wildtype, $\mathrm{KDM}_{4} \mathrm{~A}^{19}$. $2 \mathrm{OG}$ analogs have been shown to restore the activity of clinically observed variants of phytanoyl-CoA 2hydroxylase (PAHX), but do not enable wild-type PAHX catalysis $^{20}$.

Recently, we developed an efficient synthesis of C3/C4-substituted 2OG derivatives, some of which are naturally occurring ${ }^{21}$. These 2OG derivatives were shown to enhance and/or inhibit the catalytic activity of the human 2OG oxygenase aspartate/asparagine- $\beta$-hydroxylase $(\mathrm{AspH})^{21}$. AspH is a medicinal chemistry target for human cancer therapeutics and diagnostics $22-26$; it catalyzes the post-translational oxidation of Asp- and Asnresidues in specific macrocyclic disulfide isomers of epidermal growth factor-like domains (EGFDs) to give the $\beta$-erythro hydroxylated product (Fig. 1a) ${ }^{27-31}$.

Like AspH, human factor inhibiting the hypoxia-inducible transcription factor HIF- $\alpha$ (FIH) catalyzes the post-translational oxidation of Asp- and Asn-residues in a 2OG-dependent manner ${ }^{32-34}$, however, to give the $\beta$-threo hydroxylated product (Fig. 1b) ${ }^{35}$. FIH was initially identified as catalyzing hydroxylation of an Asn-residue in the C-terminal transactivation domain of HIF- $\alpha$ isoforms (Asn803 in HIF-1a), a reaction that suppresses HIF-mediated transcription $^{36}$. FIH also accepts substrates other than HIF- $\alpha^{37}$; in particular, FIH catalyzes post-translational hydroxylations of Aspand Asn-residues in ankyrin repeat domain proteins (e.g., $\mathrm{iKB}^{38}$, Notch $^{39}$, and ASB ${ }^{40}$ ). The current evidence suggests that FIH is biochemically more promiscuous with respect to its substrate requirements than $\mathrm{AspH}$ as it also catalyzes the hydroxylation of residues other than Asp and Asn (Fig. 1b) ${ }^{41-43}$.

The observation of AspH cosubstrate activity using C3/C4substituted 2OG derivatives raises the possibility of selective enhancement or inhibition of $2 \mathrm{OG}$ oxygenases by 2 -oxo acid derivatives. Here, we describe proof-of-principle studies with two isolated human $2 \mathrm{OG}$ oxygenases, i.e., $\mathrm{FIH}$ and $\mathrm{AspH}$, that validate this concept. We chose to work with FIH and AspH since because both accept Asp- and Asn-residues as hydroxylation substrates, they represent a challenge in terms of selectivity. The combined crystallographic and mass spectrometry (MS)-based studies with FIH and AspH demonstrate the potential for selective enhancement or inhibition of specific $2 \mathrm{OG}$ oxygenase activity by 2-oxo acid derivatives.

\section{Results}

Synthetic 2OG derivatives are FIH cosubstrates. The ability of 35 synthetic C3/C4-substituted 2OG derivatives, which were prepared via a reported procedure (Supplementary Fig. 1 and Supplementary Methods ${ }^{21}$, to sustain catalysis by purified recombinant FIH in the absence of $2 \mathrm{OG}$ was examined using an assay directly monitoring substrate depletion and product formation (i.e., a +16 Da mass shift) by solid-phase extraction (SPE) coupled to mass spectrometry (SPE-MS) ${ }^{44,45}$. Two peptides, based on the sequence of (1) the HIF-1a C-terminal transactivation domain fragment (i.e., HIF- $\left.1 \alpha_{788-822}\right)^{37}$ and (2) a "consensus" FIH substrate ankyrin repeat sequence ${ }^{46}\left(\text { i.e. } \mathrm{CA}_{1-20}\right)^{47}$, were used as FIH substrates to test for potential substratedependent differences in terms of cosubstrate analog selectivity. Initial screening assays were performed in the absence of $2 \mathrm{OG}$, but in the presence of high concentrations $(330 \mu \mathrm{M})$ of the synthetic C3/C4-substituted 2OG derivatives to facilitate the identification of those 2OG derivatives which sustain FIH catalysis.

In general, $\mathrm{FIH}$-catalyzed hydroxylation of $\mathrm{CA}_{1-20}$ appeared to be more efficient than of HIF-1 $\alpha_{788-822}$ when using $2 \mathrm{OG}$ as the control cosubstrate (Table 1, entry 1). High levels of FIH substrate hydroxylation were observed for 3-methyl-2OG (1) and 4-methyl$2 \mathrm{OG}(\mathbf{1 2})$, both of which are natural products ${ }^{48-51}$, regardless of the substrate, indicating that these $2 \mathrm{OG}$ derivatives can efficiently replace 2OG (Table 1, entries 2 and 13). NMR studies reveal that FIH converts 1 and 12 into 2-methylsuccinate with a similar efficiency to which it converts $2 \mathrm{OG}$ into succinate (Fig. 1c-d, Supplementary Figs. 2-4). FIH-catalyzed substrate hydroxylation was clearly apparent using other $2 \mathrm{OG}$ derivatives, i.e., 2, 3, 13-16, 22, and 28 (Entries 3, 4, 14-16, 23, and 29); however, in all these cases, conversions were lower compared to 2OG, 1 or 12 (Table 1).

The results reveal that FIH activity decreases with both substrates with the increasing length of the 2OG C3/C4-alkyl substituent (i.e., $\mathrm{Me}>\mathrm{Et}>\mathrm{Pr}$ ). The cosubstrate activity, albeit at low levels, for $\mathbf{2 2}$ and $\mathbf{2 8}$ is remarkable as their cyclic/aromatic carbon scaffolds deviate substantially from the acyclic 2OG carbon scaffold (Entries 23 and 29). Under these conditions, none of the analogs appeared to promote HIF-1a (HIF-1 $1 \alpha_{788-822}$ ) hydroxylation relative to ankyrin $\left(\mathrm{CA}_{1-20}\right)$ hydroxylation, suggesting the use of $2 \mathrm{OG}$ derivatives to modulate $\mathrm{FIH}$ activity with respect to particular substrates may be challenging (see below). All the other 2OG derivatives investigated did not show cosubstrate activity under the reaction conditions, in accord with the lack of FIH cosubstrate activity of the 2OG derivative 19, reported using an LC-MS assay ${ }^{52}$.

Comparison of these initial results for FIH with those reported for AspH ${ }^{21}$ revealed the potential of selectively promoting FIH and/ or AspH catalysis by using specific $2 \mathrm{OG}$ derivatives as cosubstrates. For example, the $2 \mathrm{OG}$ derivatives 2,3 , and 13-15 are efficient cosubstrates for $\mathrm{FIH}$, but not for AspH, whereas 35 and 29-33 showed activity with AspH, but not with FIH (Table 1) ${ }^{21}$. Notably, 3-methyl-2OG (1) is an efficient cosubstrate for both FIH and $\mathrm{AspH}$ (Table 1, entry 2), whereas its isomer 4-methyl-2OG (12) is apparently more efficient in maintaining FIH compared with $\mathrm{AspH}$ activity (Table 1, entry 13). 4-Carboxyphenylglyoxylic acid (28) is an efficient AspH cosubstrate but shows only low FIH activity with $\mathrm{CA}_{1-20}$ and none with HIF-1 $\alpha_{788-822}$ as a substrate (Table 1, entry 29). Note that our initial screening conditions differed when investigating $\mathrm{FIH}$ and $\mathrm{AspH}$ cosubstrate activity; i.e., the enzyme and substrate concentrations were different $(0.15 \mu \mathrm{M}$ and $5.0 \mu \mathrm{M}$ for FIH; $0.1 \mu \mathrm{M}$ and $2.0 \mu \mathrm{M}$ for AspH) and different buffers were used (FIH: $50 \mathrm{mM}$ Tris, $50 \mathrm{mM} \mathrm{NaCl}, \mathrm{pH}$ 7.5; AspH: $50 \mathrm{mM}$ 
a

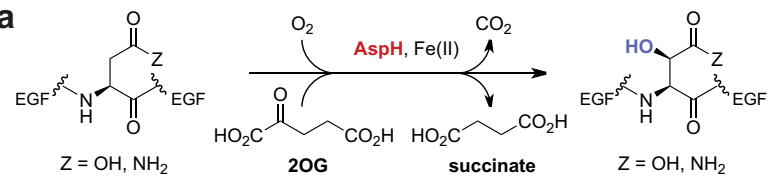

C<smiles>CC(CC(=O)O)C(=O)OCC(=O)C(C)C(=O)O</smiles>

e

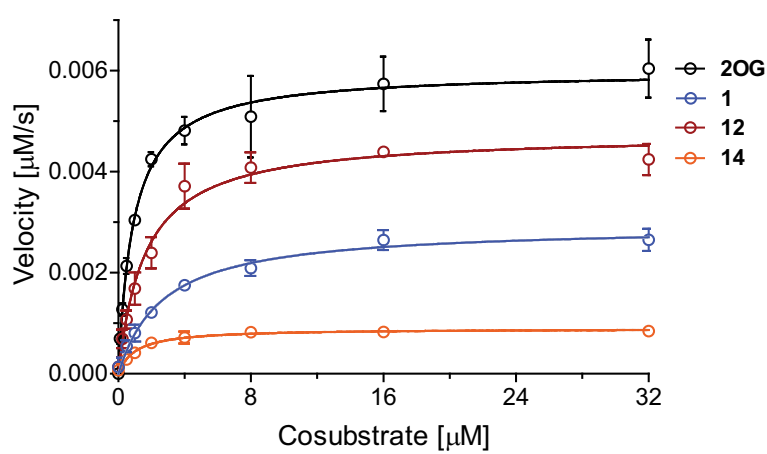

g

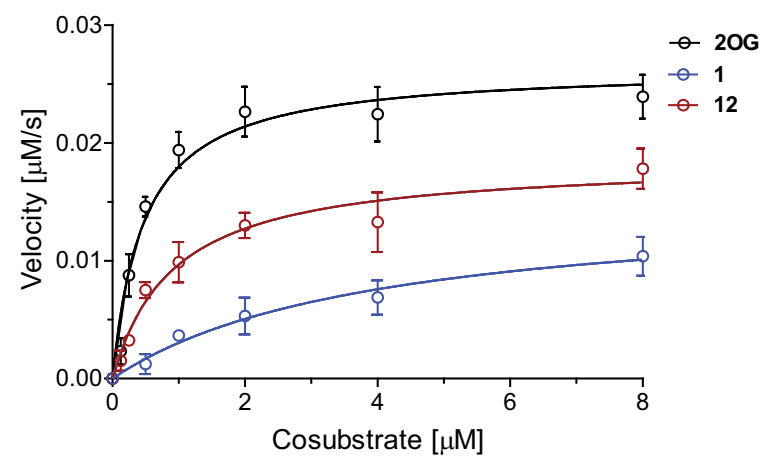

b

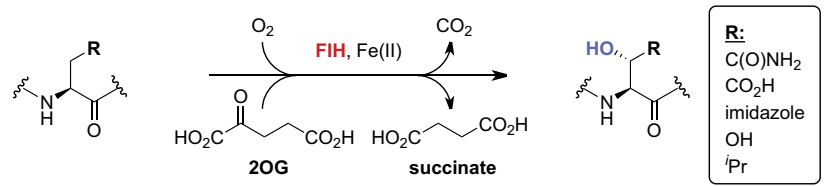

d

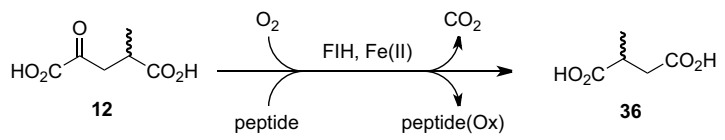

f

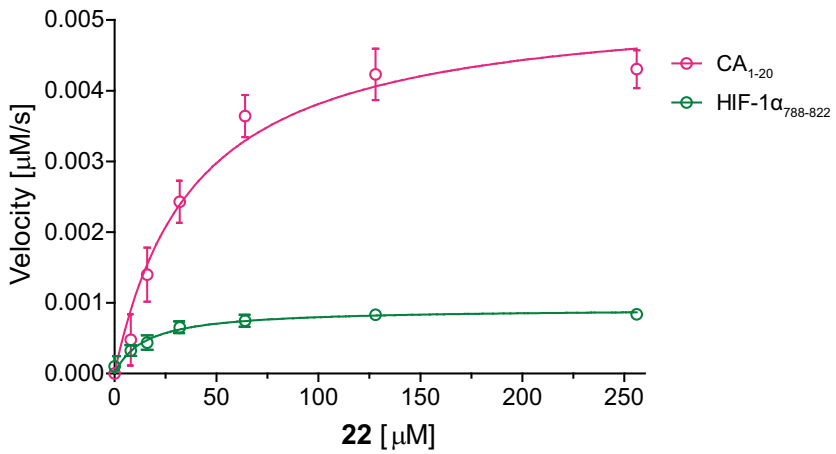

h

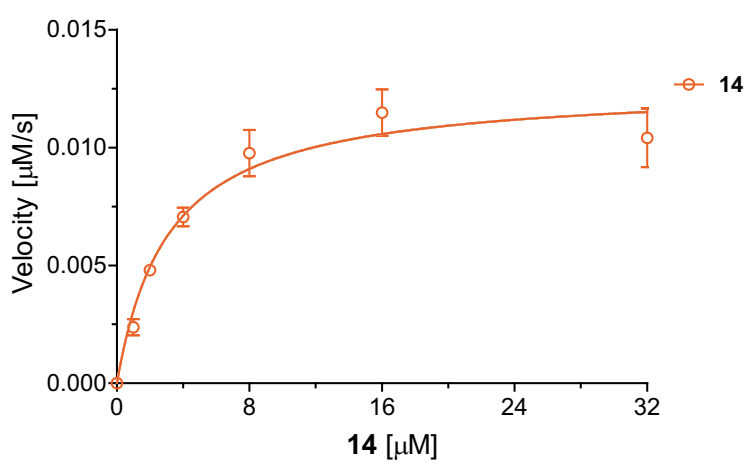

Fig. 1 Comparison of AspH and FIH catalysis and steady-state kinetic parameters for FIH-catalyzed oxidative decarboxylation of 20G derivatives from initial hydroxylation rates. a AspH and $\mathbf{b} \mathrm{FIH}$ catalyze the post-translational $\mathrm{C} 3$ hydroxylation of Asn- and Asp-residues. FIH also catalyzes the oxidation of other residues, i.e., His-, Leu- and Ser-residues 41,42 ; c and d FIH catalyzes the oxidative decarboxylation of c 3-methyl-2OG (1) and d 4-methyl-2OG (12) to give 2-methylsuccinate (36); e $K_{m}^{\text {app }}$ of FIH for $2 \mathrm{OG}$ (black), $\mathbf{1}$ (blue), $\mathbf{1 2}$ (red), and $\mathbf{1 4}$ (orange) using HIF-1 $\alpha_{788-822}$ as a substrate; $\mathbf{f} K_{m}^{\text {app }}$ of FIH for $\mathbf{2 2}$ using HIF$1 \alpha_{788-822}$ (green) or $\mathrm{CA}_{1-20}$ (pink) as a substrate; $\mathbf{g} K_{m}^{\text {app }}$ of FIH for $2 \mathrm{OG}$ (black), $\mathbf{1}$ (blue), and $\mathbf{1 2}$ (red) using $\mathrm{CA}_{1-20}$ as a substrate; $\mathbf{h} K_{m}^{\text {app }}$ of FlH for $\mathbf{1 4}$ (orange) using $\mathrm{CA}_{1-20}$ as a substrate. FIH assays were performed as described in the Methods section, data are shown as the mean of three independent runs ( $n=3$; mean \pm standard deviation, SD). The peptide hydroxylation rates are shown in Supplementary Figs. 5 and 6, source data are provided as a Source Data file.

HEPES, pH 7.5); these differences might, in part, reflect observed differences in activity with the $2 \mathrm{OG}$ derivatives. We, therefore, carried out more detailed kinetic FIH studies to better compare the results with those reported for $\mathrm{AspH}^{21}$.

Kinetic analyses. To quantify FIH activity with the 2OG derivatives, kinetic analyses of selected compounds (i.e., 2OG, 1, 12, 14, and 22) were performed using HIF- $1 \alpha_{788-822} 37$ and $\mathrm{CA}_{1-20} 47$ (Fig. 1e-h; Supplementary Figs. 5 and 6). Maximum velocities $\left(v_{\max }^{\mathrm{app}}\right)$ and Michaelis constants $\left(K_{m}^{\mathrm{app}}\right)$ were determined using $2 \mathrm{OG}$ as a control. The $v_{\max }^{\mathrm{app}}$-values reveal that the FIH-catalyzed hydroxylation of $\mathrm{CA}_{1-20}$ is faster than that of HIF- $1 \alpha_{788-822}$ for all the cosubstrates analyzed, including $2 \mathrm{OG}$ (Table 2, entry 1).
The FIH $K_{m}^{\mathrm{app}}$-values for $2 \mathrm{OG}$ are lower than those reported using different assays $(25-110 \mu \mathrm{M})^{37,53}$, but in the range of those determined for other human $2 \mathrm{OG}$ oxygenases using SPE-MS assays $(0.5-5 \mu \mathrm{M})^{27,54,55}$, likely reflecting the high sensitivity of SPE-MS assays. The FIH $K_{m}^{\text {app }}$-value for 4-methyl-2OG (12) is similar to that of 2OG, indicating a similar affinity; by contrast, the FIH $K_{m}^{\text {app }}$-value for $\mathbf{1}$, the 3-methyl regioisomer of $\mathbf{1 2}$, is higher (Table 2). Increasing the size of the alkyl substituent at C4 from methyl (12) to ethyl (14) results in a higher $K_{m}^{\text {app }}$-value when using $\mathrm{CA}_{1-20}$, whereas only a marginal effect on its $K_{m}^{\text {app }}$-value was observed when using HIF$1 \alpha_{788-822}$. In general, the FIH $K_{m}^{\mathrm{app}}$-values for $2 \mathrm{OG}, 1,12$, and 14 are in the same range $(\sim 0.5$ to $\sim 4.0 \mu \mathrm{M}$; Table 2 , entries $1-4)$, whereas the $K_{m}^{\mathrm{app}}$-values are substantially higher for $22(15$ and $39 \mu \mathrm{M}$, respectively; Table 2, entry 5). All the determined 


\section{Table 1 Synthetic C3/C4-substituted 20G derivatives sustain FIH catalysis in the absence of 20G.}

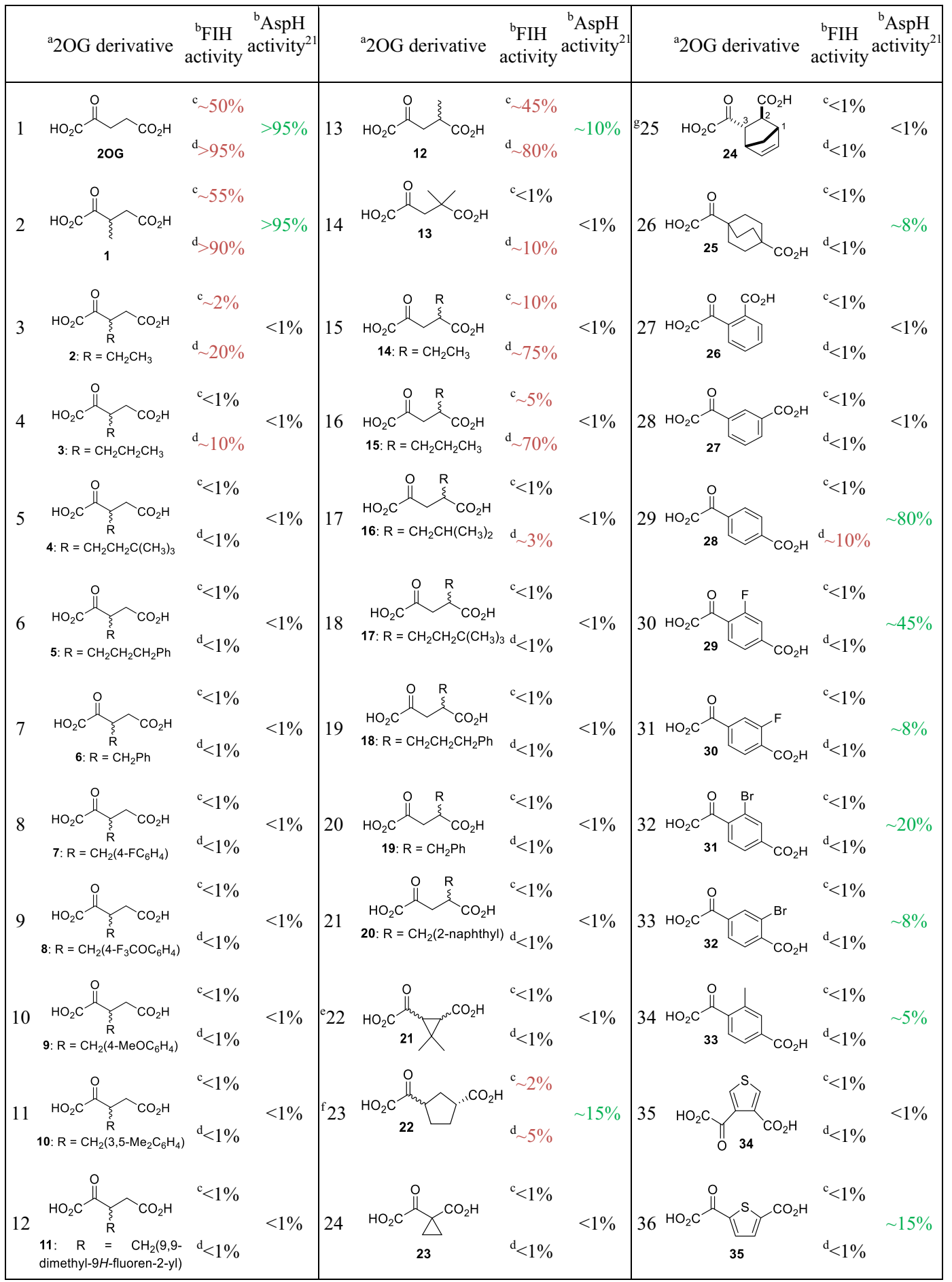

aThe synthetic C3/C4-substituted $2 \mathrm{OG}$ derivatives were prepared via a reported procedure (Supplementary Fig. 1) ${ }^{21}$, chiral $2 \mathrm{OG}$ derivatives were prepared as racemic mixtures. FIH and AspH activities for those $20 \mathrm{O}$ derivatives acting as cosubstrates are in red and green, respectively.

bSPE-MS FIH turnover assays were performed using $0.15 \mu \mathrm{M} \mathrm{FIH}, 5.0 \mu \mathrm{M}$ substrate peptide (HIF-1 $\alpha_{788-822} 37$ or CA $1-2047$ ), $50 \mu \mathrm{M} \mathrm{Fe}(\mathrm{II}), 330 \mu \mathrm{M} 20 \mathrm{G}$ or $20 \mathrm{G}$ derivative in buffer ( $50 \mathrm{mM}$ Tris, $50 \mathrm{mM}$ $\mathrm{NaCl}, \mathrm{pH} 7.5,20^{\circ} \mathrm{C}$ ). SPE-MS AspH turnover assay conditions ${ }^{21}$ : $0.1 \mu \mathrm{M} \mathrm{AspH}, 2.0 \mu \mathrm{M}$ substrate peptide (hFX-CP $\left.101-119\right), 50 \mu \mathrm{M} \mathrm{Fe}(\mathrm{II}), 330 \mu \mathrm{M} 2 \mathrm{OG}$ or $20 \mathrm{O}$ derivative in reaction buffer ( $50 \mathrm{mM} \mathrm{HEPES}$, $\mathrm{pH} 7.5,20^{\circ} \mathrm{C}$ ). \%-Substrate conversion is shown for $t=15 \mathrm{~min}$

cUsing HIF-1 $1 \alpha_{788-822 .}$

dUsing $\mathrm{CA}_{1-20}$.

${ }^{\mathrm{e}}$ Mixture of racemic diastereomers, $\mathrm{dr}$ (cis:trans) $=2.5: 1$

${ }^{\mathrm{f}}$ Mixture of diastereomers, $\mathrm{dr}$ (cis:trans) $=1: 1$.

$\mathrm{g}( \pm)$-(2-exo,3-endo)-diastereomer. 
Table 2 Comparison of the steady-state kinetic parameters of FIH for 20 G and the synthetic 20G derivatives 1, 12, 14, and 22 with those reported for AspHa,b.

\begin{tabular}{|c|c|c|c|c|c|c|}
\hline & 2OG derivative & 2OG oxygenase & $v_{\max }^{\operatorname{app}}\left[\mathrm{nM} \cdot \mathrm{s}^{-1}\right]$ & $k_{\text {cat }}^{\text {app }}\left[\mathrm{s}^{-1}\right]$ & $K_{\mathrm{m}}^{\mathrm{app}}[\mu \mathrm{M}]$ & $k_{\mathrm{cat}} / K_{\mathrm{m}}\left[\mathrm{mM}^{-1} \cdot \mathrm{s}^{-1}\right]$ \\
\hline \multirow{3}{*}{1} & & FIH (HIF-1 $\left.\alpha_{788-822}\right)$ & $6.2 \pm 0.1$ & $0.04 \pm 0.01$ & $0.8 \pm 0.1$ & $47.6 \pm 12.5$ \\
\hline & $\mathrm{HO}_{2} \mathrm{C}$ & $\mathrm{FIH}\left(\mathrm{CA}_{1-20}\right)$ & $26.4 \pm 1.2$ & $0.18 \pm 0.01$ & $0.5 \pm 0.1$ & $383.0 \pm 68.6$ \\
\hline & & $\mathrm{AspH}^{21}$ & $15.0 \pm 0.9$ & $0.17 \pm 0.03$ & $1.3 \pm 0.3$ & $129.8 \pm 32.3$ \\
\hline \multirow{3}{*}{2} & & FIH (HIF-1 $\left.\alpha_{788-822}\right)$ & $3.0 \pm 0.1$ & $0.02 \pm 0.01$ & $2.5 \pm 0.3$ & $8.1 \pm 4.2$ \\
\hline & & $\mathrm{FIH}\left(\mathrm{CA}_{1-20}\right)$ & $15.1 \pm 2.2$ & $0.10 \pm 0.02$ & $4.0 \pm 1.3$ & $25.2 \pm 9.2$ \\
\hline & & $\mathrm{AspH}^{21}$ & $6.9 \pm 0.2$ & $0.08 \pm 0.01$ & $0.27 \pm 0.04$ & $296.3 \pm 57.4$ \\
\hline \multirow{3}{*}{3} & & FIH (HIF-1 $\left.\alpha_{788-822}\right)$ & $4.8 \pm 0.1$ & $0.03 \pm 0.01$ & $1.5 \pm 0.2$ & $20.7 \pm 7.3$ \\
\hline & & $\mathrm{FIH}\left(\mathrm{CA}_{1-20}\right)$ & $18.6 \pm 1.0$ & $0.12 \pm 0.01$ & $0.9 \pm 0.2$ & $131.9 \pm 25.7$ \\
\hline & & $\mathrm{AspH}^{21}$ & - & - & - & - \\
\hline \multirow{3}{*}{4} & & FIH (HIF-1 $\left.\alpha_{788-822}\right)$ & $0.9 \pm 0.1$ & $0.01 \pm 0.001$ & $1.0 \pm 0.2$ & $9.7 \pm 1.8$ \\
\hline & $\mathrm{HO}_{2} \mathrm{C}$ & $\mathrm{FIH}\left(\mathrm{CA}_{1-20}\right)$ & $12.6 \pm 0.6$ & $0.08 \pm 0.01$ & $3.1 \pm 0.5$ & $27.0 \pm 5.3$ \\
\hline & & $\mathrm{AspH}^{21}$ & - & - & - & - \\
\hline \multirow{3}{*}{$5^{\mathrm{c}}$} & & FIH (HIF-1 $\left.1 \alpha_{788-822}\right)$ & $0.9 \pm 0.1$ & $0.01 \pm 0.001$ & $14.8 \pm 2.6$ & $0.7 \pm 0.1$ \\
\hline & & FIH $\left(\mathrm{CA}_{1-20}\right)$ & $5.3 \pm 0.3$ & $0.04 \pm 0.01$ & $39.0 \pm 6.4$ & $1.0 \pm 0.3$ \\
\hline & & $\mathrm{AspH}^{21}$ & - & - & - & - \\
\hline \multirow{3}{*}{6} & & FIH (HIF-1 $\left.\alpha_{788-822}\right)$ & - & - & - & - \\
\hline & & FIH $\left(\mathrm{CA}_{1-20}\right)$ & - & - & - & - \\
\hline & & $\mathrm{AspH}^{21}$ & $4.0 \pm 0.3$ & $0.04 \pm 0.01$ & $61.9 \pm 11.0$ & $0.7 \pm 0.2$ \\
\hline
\end{tabular}

a Mean of three independent runs $(n=3$; mean \pm SD)

bMaximum velocities $\left(v_{\max }^{\text {app }}\right)$, Michaelis constants $\left(K_{m}^{\text {app }}\right)$, turnover numbers $\left(k_{\mathrm{cat}}^{\text {app }}\right)$, and specificity constant $\left(k_{\mathrm{cat}} / K_{m}\right)$ were determined as described in the Methods section using $0.15 \mu \mathrm{M} F \mathrm{FI}$ and $5.0 \mu \mathrm{M}$

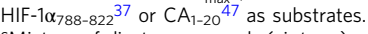

cMixture of diastereomers, $\mathrm{dr}$ (cis:trans) =1:1.

$K_{m}^{\text {app }}$-concentrations are in the range of reported physiological 2OG levels in human plasma $(9-12 \mu \mathrm{M} 2 \mathrm{OG})^{56}$, but lower than reported 2 OG concentrations in healthy cells $(\sim 1 \mathrm{mM})^{57,58}$.

An active site titration, performed using $N$-oxalyl-Dphenylalanine $^{52}$ (NOFD) as an inhibitor, revealed the apparent quantitative activity of FIH (Supplementary Fig. 7). Based on the concentration of active sites, turnover numbers (catalytic constants, $\left.k_{\text {cat }}^{\text {app }}\right)$ and specificity constants $\left(k_{\text {cat }} / K_{m}\right)$ were calculated. The $k_{\mathrm{cat}}^{\mathrm{app}}$-values decrease in the order of $2 \mathrm{OG}>12>1>14$ $\geq 22$ (Table 2). The FIH $k_{\text {cat }} / K_{m}$-values for the 2OG derivatives are higher when $\mathrm{CA}_{1-20}$ was used instead of HIF-1 $\alpha_{788-822}$. The highest FIH $k_{\text {cat }} / K_{m}$-value was observed for 2OG when using $\mathrm{CA}_{1-20}\left(\sim 383 \mathrm{mM}^{-1} \mathrm{~s}^{-1}\right.$; Table 2 , entry 1$)$, which is about eightfold higher than the value for 2OG when using HIF$1 a_{788-822}\left(\sim 48 \mathrm{mM}^{-1} \mathrm{~s}^{-1}\right.$; Table 2, entry 1$)$ and at least one order of magnitude higher than the values observed for the 2OG derivatives investigated, with the notable exception of the $k_{\text {cat }} / K_{m}$-value with 12 using $\mathrm{CA}_{1-20}$, compared with which it is approximately threefold higher $\left(\sim 132 \mathrm{mM}^{-1} \mathrm{~s}^{-1}\right.$; Table 2 , entry $3)$. The results reveal that the FIH $k_{\mathrm{cat}} / K_{m}$-values for the $2 \mathrm{OG}$ derivatives vary, to some extent, with the nature of the substrate. Considering the high number of reported FIH substrates, this 
a
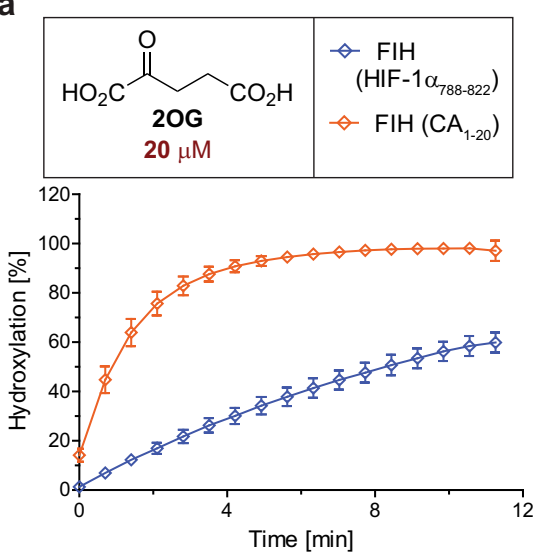

d
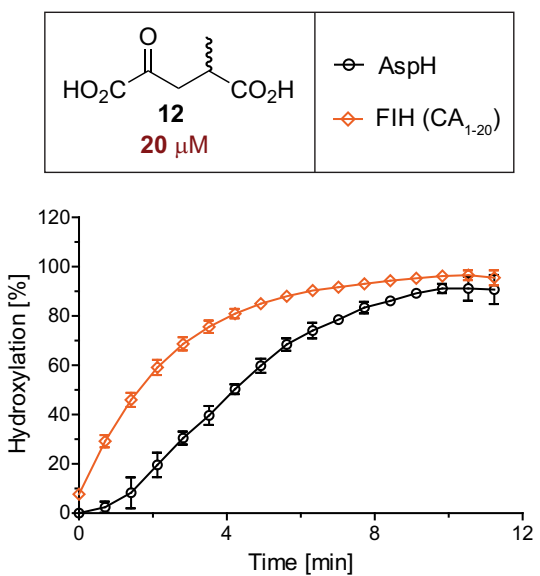

b
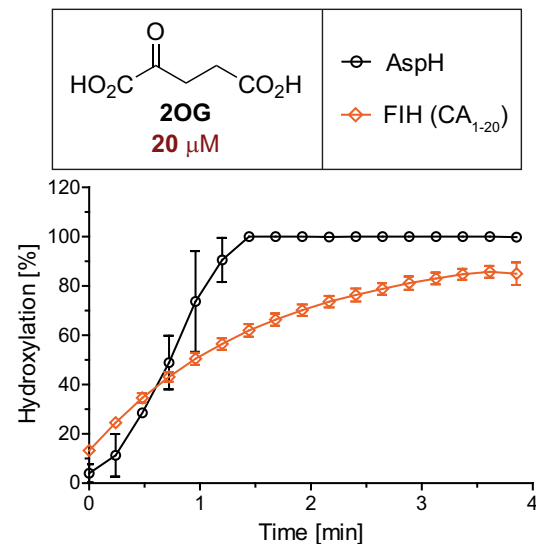

e
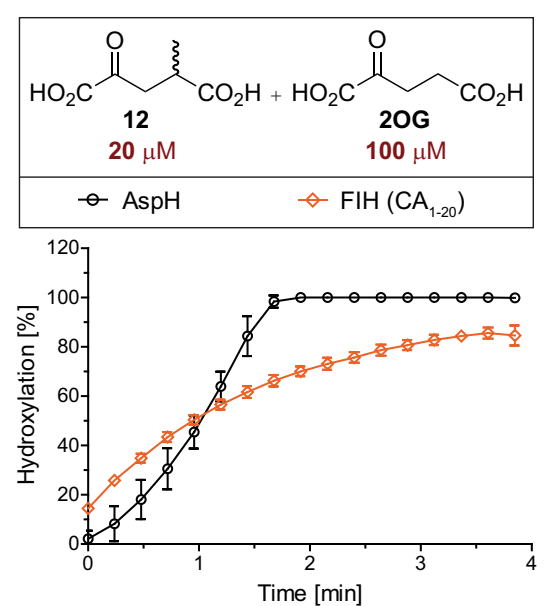
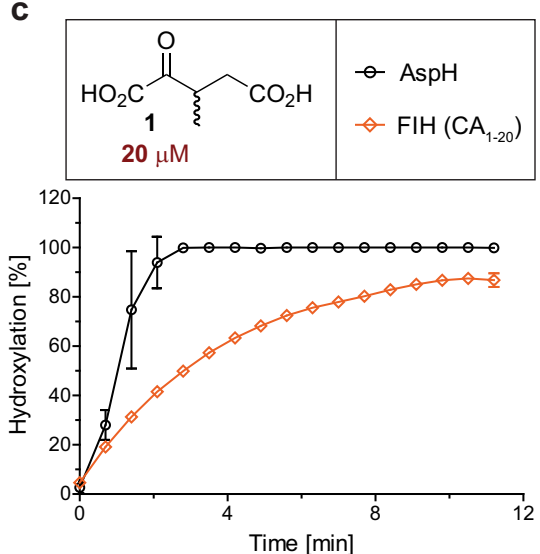

f
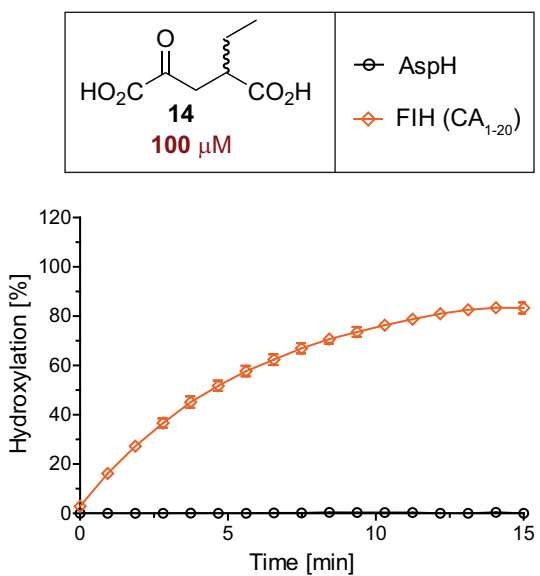

Fig. 2 20G derivatives selectively sustain the catalytic activities of 20 G oxygenases in vitro. Assays were performed as described in the Methods section using $0.15 \mu \mathrm{M}$ of each $2 \mathrm{OG}$ oxygenase (diamond: $\mathrm{FIH}$, circle: $\mathrm{AspH}$ ) and $5.0 \mu \mathrm{M}$ of each substrate (blue: $\mathrm{HIF}^{-1} \alpha_{788-822}{ }^{37}$; orange: $\mathrm{CA}_{1-20}{ }^{47}$; black: hFX-EGFD1 $86-124-4 S^{27,31}$, AspH substrate) in the presence of $100 \mu \mathrm{M}$ LAA, $10 \mu \mathrm{M} F A$, and the indicated concentration of cosubstrate in buffer (50 mM Tris, $50 \mathrm{mM} \mathrm{NaCl}, \mathrm{pH}$ 7.5). Mean of three independent runs ( $n=3$; mean $\pm \mathrm{SD}$ ). Measurement times were normalized to the first sample injection analyzed after the addition of the $20 \mathrm{G}$ oxygenase to the Substrate Mixture ( $t=0 \mathrm{~min}$ ), by which time low levels of hydroxylation were manifest. Cosubstrates: a $20 \mu \mathrm{M}$ 20G; b $20 \mu \mathrm{M}$ 20G; c $20 \mu \mathrm{M}$ 1; d $20 \mu \mathrm{M}$ 12; e $20 \mu \mathrm{M} 12$ and $100 \mu \mathrm{M} 20 \mathrm{G}$; f $100 \mu \mathrm{M}$ 14. Source data are provided as a Source Data file.

observation suggests that for some substrates, the $k_{\text {cat }} / K_{m}$-values of FIH for $2 \mathrm{OG}$ derivatives might actually be higher than for 2OG. They also reveal that the $k_{\text {cat }} / K_{m}$-values of FIH for the $2 \mathrm{OG}$ derivatives might be higher for a specific substrate (i.e., when using 12 and $\mathrm{CA}_{1-20}$; Table 2, entry 3 ) than the $k_{\text {cat }} / K_{m}$-values of $\mathrm{FIH}$ for the natural cosubstrate $2 \mathrm{OG}$ when using different substrates (i.e., HIF-1 $\alpha_{788-822}$; Table 2, entry 1).

The FIH $k_{\text {cat }} / K_{m}$-values for the $2 \mathrm{OG}$ derivatives are in the range of those reported for $\mathrm{AspH}$, which were also obtained using SPE-MS assays (Table 2) 21 . Notably, the FIH $k_{\text {cat }} / K_{m}$-value for 2 OG with $\mathrm{CA}_{1-20}$ is about threefold higher than the one of $\mathrm{AspH}$ for $2 \mathrm{OG}$ $\left(\sim 383\right.$ and $\sim 130 \mathrm{mM}^{-1} \mathrm{~s}^{-1}$, respectively; Table 2$)$. By contrast, the FIH $k_{\text {cat }} / K_{m}$-value for 1 with $\mathrm{CA}_{1-20}$ is an order of magnitude lower than that for AspH ( 25 and $\sim 296 \mathrm{mM}^{-1} \mathrm{~s}^{-1}$, respectively; Table 2). The FIH $k_{\text {cat }} / K_{m}$-values for 22 are similar to the reported one of AspH for $28\left(\sim 1 \mathrm{mM}^{-1} \mathrm{~s}^{-1} \text {; Table 2, entries } 5 \text { and } 6\right)^{21}$, which, however, is not an efficient FIH cosubstrate.

FIH and AspH competition studies. Comparisons of the effects of the synthetic 2OG derivatives on FIH catalysis with their reported effect on $\mathrm{AspH}$ catalysis $^{21}$ indicated their potential to selectively enhance FIH or AspH activity. However, the FIH and $\mathrm{AspH}$ data were obtained under different conditions (different enzyme/(co-)substrate/cofactor concentrations, enzyme/substrate ratios, and buffers), possibly perturbing the results of their direct comparison. It is an advantage of MS-based turnover assays that the hydroxylation of different substrate peptides can be monitored in parallel in a single reaction vessel. Thus, the FIHcatalyzed hydroxylation of two different substrates as well as FIHand AspH-catalyzed substrate hydroxylations were assessed simultaneously in the same vessel, complementing prior kinetic and turnover studies using a single isolated enzyme and substrate.

An equimolar mixture of the HIF- $1 \alpha_{788-822} 37$ (blue) and $\mathrm{CA}_{1-20} 47$ (orange) peptides in a single reaction vessel was submitted to FIHcatalyzed modification at saturating $2 \mathrm{OG}$ and $\mathrm{Fe}$ (II) concentrations (Fig. 2a). The observed turnovers reflect the results of the kinetic experiments with individual substrates (Table 2). In agreement with an approximate eightfold difference observed in the $\mathrm{FIH}$ $k_{\text {cat }} / K_{m}$-values for $2 \mathrm{OG},>90 \%$ conversion of $\mathrm{CA}_{1-20}$ was observed after $5 \mathrm{~min}$, while only $\sim 60 \%$ conversion of HIF- $1 \alpha_{788-822}$ was observed after $12 \mathrm{~min}$. This observation raises the possibility of the use of $2 \mathrm{OG}$ derivatives for selective enhancement of the oxidation of specific sets of substrates for a $20 \mathrm{OG}$ oxygenase.

Next, an equimolar amount of the FIH substrate $\mathrm{CA}_{1-20}$ and the $\mathrm{AspH}$ substrate $\mathrm{hFX}-\mathrm{EGFD} 1_{86-124}-4 \operatorname{Ser}(5.0 \mu \mathrm{M}$ each), the latter based on the sequence of the reported AspH substrate 
human coagulation factor $\mathrm{X}^{27,31,59}$, was submitted to equimolar amounts of FIH and $\mathrm{AspH}(0.15 \mu \mathrm{M}$ each) in the presence of $2 \mathrm{OG}(20 \mu \mathrm{M})$ and $\mathrm{Fe}(\mathrm{II})(10 \mu \mathrm{M})$ in a single reaction vessel. The presence of two 2OG oxygenases did not result in substantial peptide ionization suppression and high-quality time course data were obtained: $>95 \%$ AspH-catalyzed hFX-EGFD1 $1_{86-124}-4 \mathrm{Ser}$ hydroxylation was observed within about $1 \mathrm{~min}$, whereas $>80 \%$ FIH-catalyzed $\mathrm{CA}_{1-20}$ hydroxylation was observed after $4 \mathrm{~min}$ (Fig. 2b). By contrast to the analysis of the FIH and AspH kinetic parameters for $2 \mathrm{OG}$ with individual isolated enzymes (Table 2, entry 1), the results reveal that, when using $2 \mathrm{OG}$ as a cosubstrate under the tested conditions, $\mathrm{AspH}$ catalysis is more efficient than FIH catalysis. This might reflect the dimeric nature of FIH in solution ${ }^{60}$ and/or the different assay conditions used to obtain the kinetic parameters for isolated $\mathrm{FIH}$ and $\mathrm{AspH}$; for example, a shorter substrate than the hFX-EGFD1 $1_{86-124}-4$ Ser peptide was used when determining the AspH kinetic parameters ${ }^{21}$.

In a similar single vessel experiment, an approximately twofold decrease in FIH efficiency was observed when 2OG was substituted for $\mathbf{1}(20 \mu \mathrm{M})$, keeping other reaction parameters same, i.e., $>80 \% \mathrm{CA}_{1-20}$ hydroxylation was observed after about $8 \mathrm{~min}$ rather than $4 \mathrm{~min}$ (Fig. $2 \mathrm{c}$ ), whereas no detrimental effect on AspH catalysis was observed ( $>95 \%$ hFX-EGFD1 $1_{86-124}-4$ Ser hydroxylation within $\sim 2$ min Fig. $2 c$ ), in agreement with the kinetics of the individual enzymes (Table 2).

As the kinetic data with isolated $\mathrm{FIH}$ and $\mathrm{AspH}$ suggests (Table 2), using a saturating concentration of $12(20 \mu \mathrm{M})$ as a cosubstrate instead of $2 \mathrm{OG}$ or $\mathbf{1}$, reversed the reactivity profile (Fig. 2d). Thus, with 12, FIH catalysis was more efficient than AspH catalysis, reaching $\sim 90 \% \mathrm{CA}_{1-20}$ hydroxylation after $\sim 5$ min, whereas AspH-catalyzed hFX-EGFD1 $1_{86-124}$-4Ser hydroxylation reached $\sim 90 \%$ after $\sim 10 \mathrm{~min}$ in the same reaction vessel. Interestingly, the presence of both $2 \mathrm{OG}(100 \mu \mathrm{M})$ and $12(20 \mu \mathrm{M})$ in the same reaction vessel reversed this effect, i.e., AspH catalysis is faster than $\mathrm{FIH}$ catalysis (Fig. 2e). Complete AspH-catalyzed hydroxylation of hFX-EGFD1 $1_{86-124}-4$ Ser was observed after $\sim 2$ min, which is only marginally slower than in the absence of any 12 (Fig. 2b). The FIH-catalyzed hydroxylation of $\mathrm{CA}_{1-20}$ was not affected; it reaches $\sim 90 \%$ conversion in the presence of saturating $2 \mathrm{OG}$ or 12 concentrations, and also in the presence of both cosubstrates (Fig. 2e).

Comparing $\mathrm{FIH}$ and $\mathrm{AspH}$ activities in a single reaction vessel reveals that only $\mathrm{FIH}$-catalyzed hydroxylation is observed when using $2 \mathrm{OG}$ derivative $\mathbf{1 4}(100 \mu \mathrm{M})$ in the absence of 2OG (Fig. 2f). However, FIH catalysis was less efficient for $\mathbf{1 4}$ than for 2OG, 1 or 12, which were used at lower concentrations $(20 \mu \mathrm{M})$. AspHcatalyzed hydroxylation of hFX-EGFD1 $1_{86-124}-4$ Ser was not observed, in agreement with the reported lack of cosubstrate activity with $\mathbf{1 4}$ $(\text { Table } 1)^{21}$. Similarly, selective enhancement of AspH activity was observed without 2OG using the phenyl-ring containing $2 \mathrm{OG}$ derivative 28 (Supplementary Fig. 8), which showed negligible amounts of cosubstrate activity for FIH (Table 1).

Crystallography. Crystallographic studies were initiated to investigate the binding of the $2 \mathrm{OG}$ derivatives to $\mathrm{FIH}$ and to compare the results with those reported for $\mathrm{AspH}^{21}$. Initially, FIH was crystallized in the presence of $\mathrm{Zn}(\mathrm{II})$, as a substitute for $\mathrm{Fe}(\mathrm{II})$, and in the presence of the C3-alkyl-substituted 2OG derivatives $\mathbf{1}$ and $\mathbf{3}$. In the absence of substrate, the FIH complexes crystallized in the $P 4_{1} 2{ }_{1} 2$ space group (FIH:1, $2.29 \AA$ resolution; FIH:3, $2.18 \AA$ resolution; Supplementary Figs. 9-12). The structures were solved by molecular replacement $(\mathrm{MR})$ using a reported FIH structure (PDB ID: $1 \mathrm{H} 2 \mathrm{~K})^{61}$ as a search model. The superimposition of both structures with the reported FIH:2OG:HIF-1 $1 a_{786-826}$ structure (PDB ID: $1 \mathrm{H} 2 \mathrm{~L})^{61}$ reveals that the conformation of $2 \mathrm{OG}$ does not substantially change upon introducing substituents at $\mathrm{C} 3$, albeit the conformation of the C3-C4 ethylene unit of $\mathbf{1}$ and $\mathbf{3}$ slightly differs from that of $2 \mathrm{OG}$ (Fig. 3a). The C3-alkyl substituents apparently align to interact with the hydrophobic sidechains of Leu186 and Leu188.

FIH co-crystallized with $1, \mathrm{Zn}$, and $\mathrm{CA}_{1-20}$ in the $P 4_{1} 2{ }_{1} 2$ space group (FIH:1: $\mathrm{CA}_{1-20}, 2.01 \AA$ resolution; Supplementary Fig. 13). All FIH:substrate structures were solved by MR using a reported FIH structure (PDB ID: $1 \mathrm{H} 2 \mathrm{~K})^{61}$ as a search model. Superimposition of the FIH:1:CA $\mathrm{CA}_{1-20}$ structure with the reported FIH:2OG:HIF-1 $\alpha_{786-826}$ (PDB ID: 1H2L; Fig. 3b) ${ }^{61}$ and the FIH:1 structures (Fig. 3c) reveals that the conformation of 1 does not substantially change upon substrate binding (Supplementary Fig. 14). The nature of the FIH substrate does not seem to influence the conformation of $\mathbf{1}$, as revealed by co-crystallization with a fragment of the ankyrin repeat-containing validated human FIH substrate tankyrase-2 ${ }^{62}$ (FIH:1:TNKS2 $691-710 ; 2.01 \AA$ resolution; Supplementary Figs. 15-17).

$\mathrm{FIH}$ was also crystallized in the presence of $\mathrm{CA}_{1-20}$ and the $\mathrm{C} 4$ alkyl-substituted 2OG derivatives 14 and 15 (FIH:14:CA $\mathrm{CA}_{1-20}$, $2.21 \AA$ resolution; FIH:15: $\mathrm{CA}_{1-20}, 1.76 \AA$ resolution; Supplementary Figs. 18-21). The conformations of 14 and 15 were very similar to that of $2 \mathrm{OG}$ with their C4-alkyl substituents facing towards the hydrophobic Leu186 and Leu188 sidechains (Fig. 3d), as observed for the C3-alkyl 2OG derivatives. The observation that the conformations of C3- and C4-alkyl 2OG derivatives are very similar to that of $2 \mathrm{OG}$ in complex with FIH is in agreement with their similar $K_{m}^{\mathrm{app}}$-values (Table 2). The difference in $k_{\text {cat }}^{\text {app }}$ is the major factor for the observed differences in the $k_{\text {cat }} / K_{m}$-values for 14 and 2OG; the lower $k_{\text {cat }}^{\text {app }}$ for 14 may reflect the lower conformational/rotational flexibility of the substituted 2OG derivatives due to stronger hydrophobic interactions with the Leu186 and Leu188 sidechains and/or the enhanced binding to and thus slower release of the corresponding alkyl-substituted succinate reaction product from $\mathrm{FIH}$.

In the FIH:22: $\mathrm{CA}_{1-20}$ structure (1.75 $\AA$ resolution; Supplementary Figs. 22-23), 22 occupies a different conformation than that observed for 2OG, possibly to compensate for its rigid cyclic carbon-backbone (Fig. 3e). The FIH $K_{m}^{\text {app }}$ for 22 reflects this conformational difference as it is about tenfold higher compared with the $K_{m}^{\text {app }}$ for 2OG, 1, and 14, regardless of the substrate employed (Table 2), indicative of a lower affinity of FIH for 22.

The hydrophobic interactions of the C3/C4-alkyl 2OG derivatives with the Leu186 and Leu188 sidechains in FIH resemble those of the C3/C4-alkyl-substituted 2OG derivatives with the Met670, Val676, and Val727 sidechains in AspH structures (Fig. $3 \mathrm{f}$ and Supplementary Figs. 24-25) 21 . In addition to differences in the $2 \mathrm{OG} \mathrm{C5}$ carboxylate binding motif, the interaction of aliphatic $\mathrm{C} 3 / \mathrm{C} 4$ substituents of the 2OG derivatives with hydrophobic residues and/ or hydrophobic pockets (as observed for $\mathrm{AspH}$ ) in the active sites of $2 \mathrm{OG}$ oxygenases, appears to influence the conformation of the $2 \mathrm{OG}$ derivatives. Although the C3/C4-alkyl 2OG derivatives were used as racemic mixtures, the crystallographic analyses reveal that, at least predominantly, the $(S)$-enantiomers of the $2 \mathrm{OG}$ derivatives bind to $\mathrm{FIH}$, potentially owing to their ability to engage in more efficient hydrophobic interactions with the hydrophobic Leu186 and Leu188 sidechains (Supplementary Figs. 10, 12, 14, 16, 19, and 21). A similar observation has been made for $\mathrm{AspH}$, however, in this case, the $(R)$-enantiomers of the C3/C4-alkyl-substituted $2 \mathrm{OG}$ derivatives engage in hydrophobic interactions with $\mathrm{AspH}^{21}$. Interestingly, the unusual geometry of the $\mathrm{AspH} F e(\mathrm{II})$-binding site $^{21,31}$, i.e., only two AspH histidine residues coordinate the $\mathrm{Fe}(\mathrm{II})$ cofactor (e.g., $\mathrm{H}_{679} \mathrm{XG}_{681} \ldots \mathrm{H}_{725}$ ) rather than the typical triad of ligands $(\mathrm{HXD} / \mathrm{E} \ldots \mathrm{H})$ found in the active site of other human $2 \mathrm{OG}$ hydroxylases including $\mathrm{FIH}\left(\mathrm{H}_{199} \mathrm{XD}_{201} \ldots \mathrm{H}_{279}\right)^{1}$, appears not to 
a

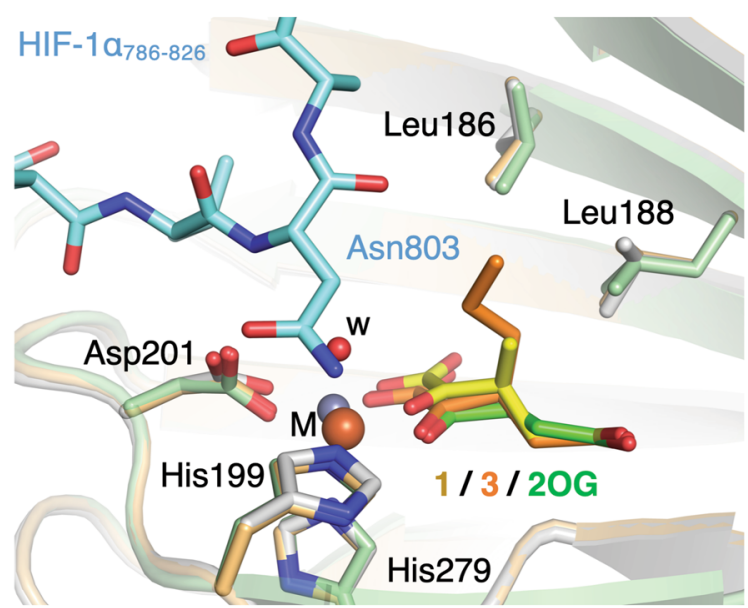

C

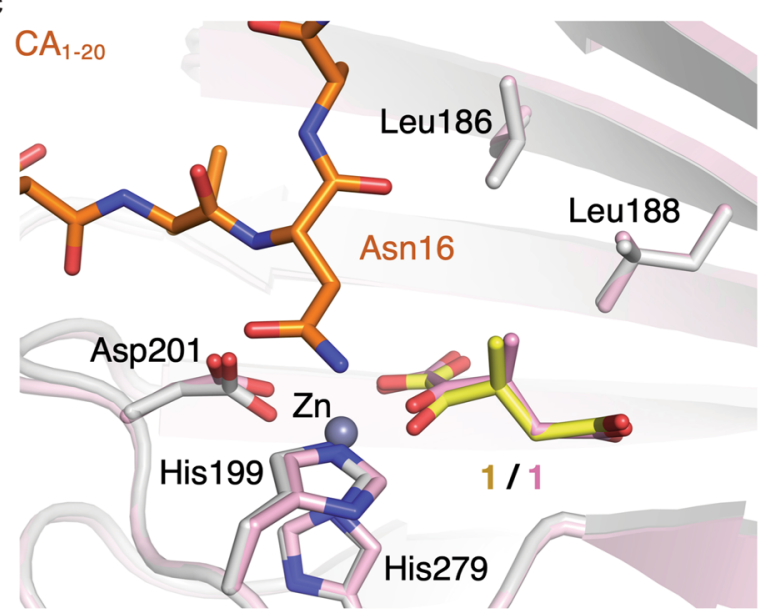

e

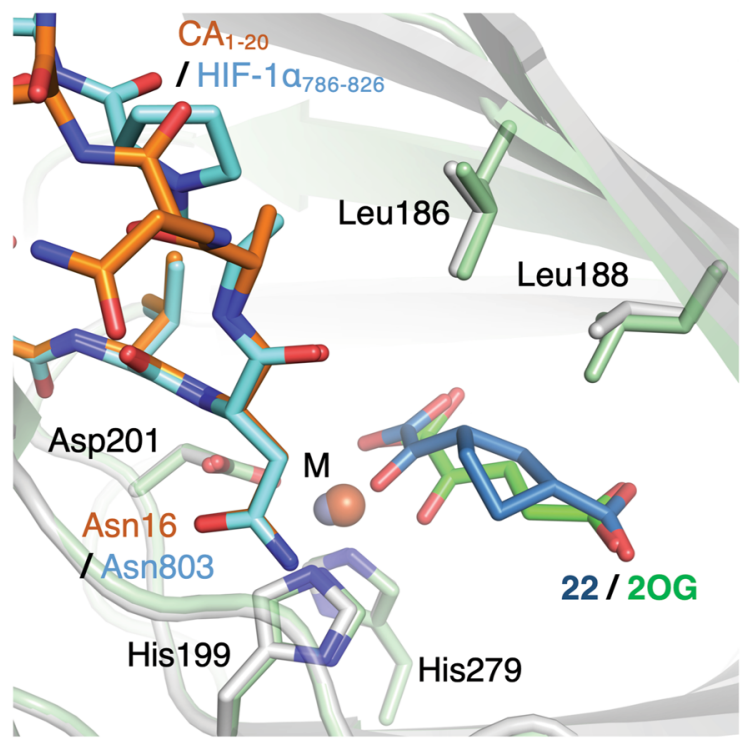

b

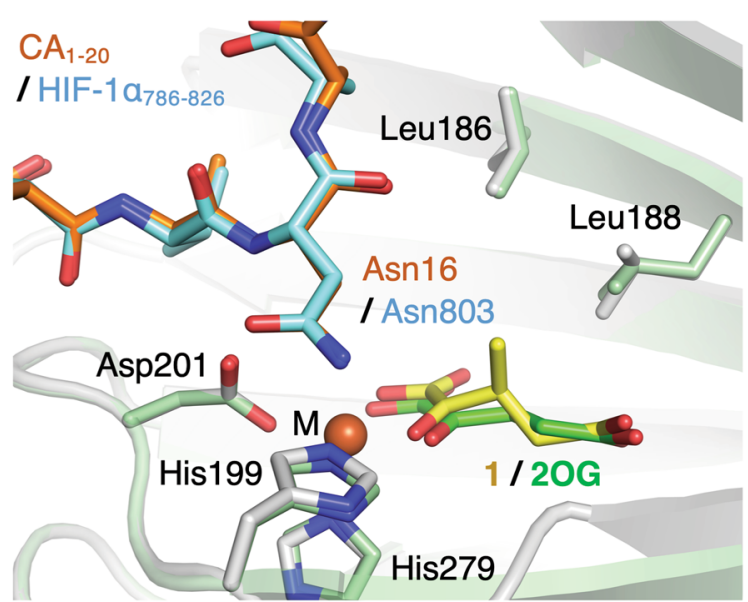

d

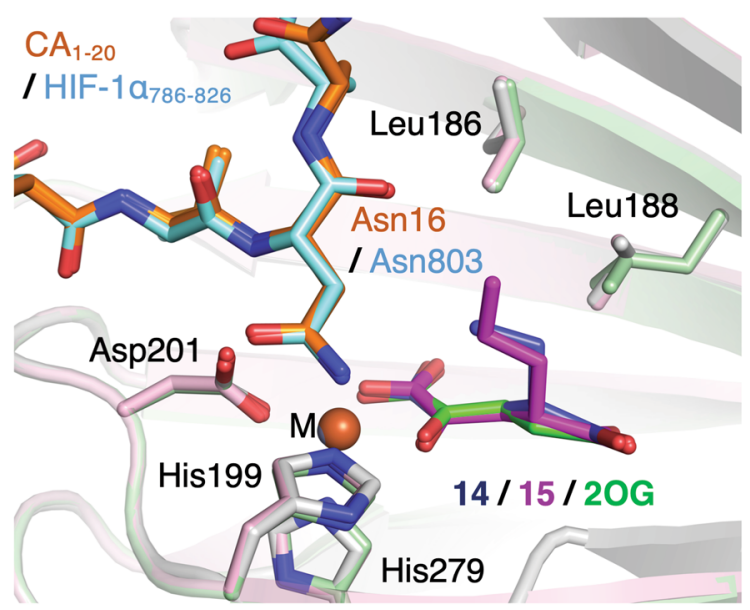

f

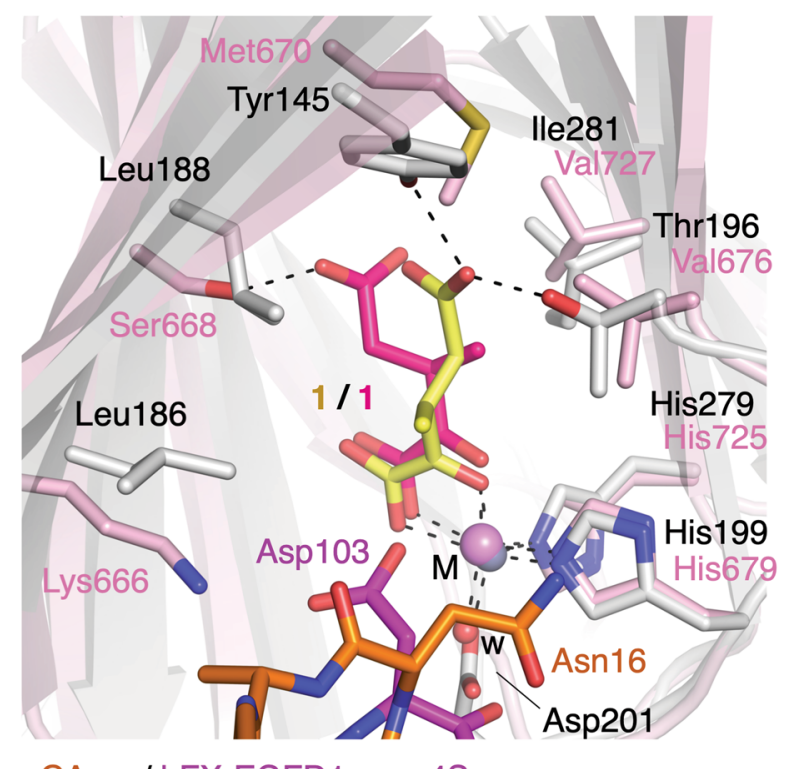

$\mathrm{CA}_{1-20} / \mathrm{hFX}-\mathrm{EGFD1} 1_{86-124}-4$ Ser directly affect $2 \mathrm{OG}$ derivative binding to $\mathrm{AspH}$ nor AspH/FIH cosubstrate selectivity based on the superimposition of AspH and FIH substrate structures complexed with 1 (Fig. 3f).

Synthetic 2OG derivatives inhibit FIH. To further investigate the utility of the 35 synthetic C3/C4-substituted 2OG derivatives for selectively modulating FIH or AspH activity, their inhibitory effect on FIH activity was investigated ${ }^{44,45}$. As the crystallographic studies revealed that the conformations of the $\mathrm{CA}_{1-20}$ and HIF- $1 \alpha_{786-826}$ peptides align well at the FIH active site (Fig. $3 b, d$, and e), i.e. the predicted binding site of the $2 \mathrm{OG}$ derivatives, the inhibition assays were performed exclusively with HIF- $1 \alpha_{788-822}{ }^{37}$ owing to its reduced reactivity, facilitating the 
Fig. 3 The synthetic $20 \mathrm{G}$ derivatives 1, 3, 14, 15, and 22 bind FIH similarly to 2OG. a Superimposition of views from the FIH:1 (FIH: gray; Zn ion: lavender blue; carbon-backbone of $\mathbf{1}$ : yellow) and $\mathrm{FIH:3} \mathrm{(FIH:} \mathrm{light} \mathrm{orange;} \mathrm{Zn}$ ion: lavender blue; carbon-backbone of 3: orange; w: water) structures with a view from the reported FIH:2OG:HIF-1 $\alpha_{786-826}$ structure (FIH: pale green; Fe ion: orange; carbon-backbone of 2OG: green; carbon-backbone of HIF-1 $\alpha_{786-826}$ : cyan;

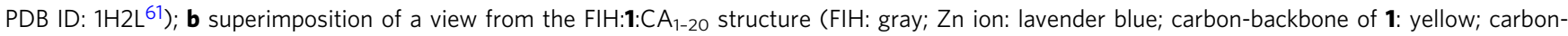
backbone of $\mathrm{CA}_{1-20}$ : orange) with one from the reported FIH:2OG:HIF-1 $\alpha_{786-826}$ structure (FIH: pale green; Fe ion: orange; carbon-backbone of 2OG: green; carbon-backbone of HIF-1 $\alpha_{786-826}$ : cyan; PDB ID: $1 \mathrm{H}_{2} \mathrm{~L}^{61}$ ); c superimposition of a view from the FIH:1 structure (FIH: light pink; $\mathrm{Zn}$ ion: lavender blue; carbon-backbone of 1: pink) with one from the $\mathrm{FIH:1}: \mathrm{CA}_{1-20}$ structure ( $\mathrm{FIH}$ : gray; $\mathrm{Zn}$ ion: lavender blue; carbon-backbone of 1: yellow; carbon-backbone of

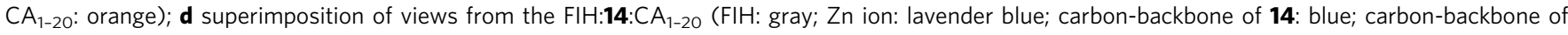
$\mathrm{CA}_{1-20}$ : orange) and $\mathrm{FIH}: 15: \mathrm{CA}_{1-20}\left(\mathrm{FIH}\right.$ : light pink; $\mathrm{Zn}$ ion: lavender blue; carbon-backbone of 15: magenta; carbon-backbone of $\mathrm{CA}_{1-20}$ : orange) structures with a view from the reported FIH:2OG:HIF-1 $\alpha_{786-826}$ structure (FIH: pale green; Fe ion: orange; carbon-backbone of 2OG: green; carbon-backbone of HIF1 $\alpha_{786-826}$ : cyan; PDB ID: $\left.1 \mathrm{H}_{2} \mathrm{~L}^{61}\right)$; e superimposition of a view from the FIH:22: $\mathrm{CA}_{1-20}$ structure $(\mathrm{FIH}$ : gray; $\mathrm{Zn}$ ion: lavender blue; carbon-backbone of 22: sky blue; carbon-backbone of $\mathrm{CA}_{1-20}$ : orange) with one from the reported FIH:2OG:HIF-1 $\alpha_{786-826}$ structure (FIH: pale green; Fe ion: orange; carbon-backbone of 2OG: green; carbon-backbone of HIF-1 $\alpha_{786-826}$ : cyan; PDB ID: $\left.1 \mathrm{H}_{2} \mathrm{~L}^{61}\right)$; $\mathbf{f}$ superimposition of a view from the FIH:1:CA $1-20$ structure (FIH: gray; $\mathrm{Zn}$ ion: lavender blue; carbon-backbone of 1: yellow; carbon-backbone of $\mathrm{CA}_{1-20}$ : orange) with one from the reported AspH:1:hFX-EGFD1 $86-124-4$ Ser structure (AspH: light pink; $\mathrm{Mn}$ ion: violet; carbon-backbone of 1: hot pink; carbon-backbone of hFX-EGFD1 $86-124$-4Ser: magenta; w: water; PDB ID: 6YYX21). Information on source data is provided in the Data availability section.

experimental set-up. Half maximum inhibitory concentrations $\left(\mathrm{IC}_{50}\right.$-values) of the $2 \mathrm{OG}$ derivatives were determined (comprehensive analysis in Supplementary Table 1), using NOFD ${ }^{52}$ as a positive inhibition control. The $\mathrm{IC}_{50}$ for NOFD $(\sim 0.2 \mu \mathrm{M}$; Table 3, entry 1) is substantially lower in the SPE-MS assay compared to a previously employed derivatization assay $\left(K_{i}^{\text {app }} \sim 93 \mu \mathrm{M}\right)^{52}$, highlighting the better sensitivity of this direct assay $(0.15 \mu \mathrm{M}$ FIH was used in the SPE-MS assay and $4.0 \mu \mathrm{M}$ FIH in the derivatization assay).

The increase in length of the 2OG C3/C4-substituent from methyl via ethyl to propyl appears to correlate with enhanced FIH inhibition (Table 3). Thus, although the C3/C4 methylsubstituted 2OG derivatives $\mathbf{1}$ and $\mathbf{1 2}$ do not inhibit FIH (Table 3, entries 2 and 7), 3-propyl-2OG (3) inhibits FIH efficiently ( $\mathrm{IC}_{50}$ $\sim 2.7 \mu \mathrm{M}$; Table 3 , entry 4 ). The potencies of the synthetic $2 \mathrm{OG}$ derivatives as a function of substituent length appear to inversely correlate with their cosubstrate efficiency (Tables 1 and 3). For example, high levels of FIH cosubstrate activity, but not FIH inhibition, were observed for $\mathbf{1}$ and $\mathbf{1 2}$ (Table 1, entries 2 and 13), whereas the C4 ethyl and propyl-substituted 2OG derivatives 14 and $\mathbf{1 5}$ are more efficient FIH cosubstrates, but less efficient FIH inhibitors compared to the corresponding C3 regioisomers 2 and 3 (Tables 1 and 3).

C4-Benzyl-2OG (19) inhibits FIH efficiently in the SPE-MS assay ( $\mathrm{IC}_{50} \sim 3.6 \mu \mathrm{M}$; Table 3 , entry 12 ), whereas only weak inhibition has been reported when employing a 2-oxo acid derivatization assay ${ }^{52}$. Notably, 19 inhibits FIH more than one order of magnitude less efficiently than the structurally related, but conformationally more rigid, NOFD (note that NOFD was used in the enantiopure form, while $\mathbf{1 9}$ was used as a racemic mixture). However, the potency of $\mathbf{1 9}$ was increased when replacing its $\mathrm{C} 4$ benzyl- with a 3-phenylpropyl-substituent $\left(\mathrm{IC}_{50}\right.$ $\sim 0.9 \mu \mathrm{M}$; Table 3, entry 11). The C4 benzyl- and C4 3phenylpropyl-substituted 2OG derivatives 19 and 18 are twoand sixfold, respectively, more efficient FIH inhibitors than their corresponding C3 regioisomers (Table 3, entries 5, 10, 11, and Supplementary Table 1). However, the potency of C3 benzylderivative 6 could be substantially enhanced when introducing a sterically bulkier $\mathrm{CH}_{2}$ (9,9-dimethyl-9H-fluoren-2-yl-substituent at $\mathrm{C} 3\left(\mathrm{IC}_{50} \sim 1.9 \mu \mathrm{M}\right.$; Table 3, entry 6).

In general, the $2 \mathrm{OG}$ derivatives inhibit FIH less efficiently than $\mathrm{AspH}$, which might, at least to some extent, reflect the higher $2 \mathrm{OG}$ concentration used in the FIH inhibition assays $(10 \mu \mathrm{M}$ $2 \mathrm{OG}$ in the FIH assays, $3 \mu \mathrm{M} 2 \mathrm{OG}$ in the AspH assays). Thus, the C4 ethyl- and propyl-substituted 2OG derivatives $\mathbf{1 4}$ and $\mathbf{1 5}$ are highly efficient $\mathrm{AspH}$ inhibitors, consistent with their reported lack of AspH cosubstrate activity ${ }^{21}$, whereas the same compounds are more than an order of magnitude less efficient in inhibiting FIH (Table 3, entries 9 and 10). Inhibition selectivity is even higher for 4,4-dimethyl-2OG (13), which efficiently inhibits AspH $\left(\mathrm{IC}_{50} \sim 0.4 \mu \mathrm{M}^{21}\right)$ but not at all FIH (Table 3, entry 8 ). This result highlights the importance of apparently minor structural modifications on inhibitor selectivity as 4-methyl-2OG (12) neither inhibits AspH nor FIH (Table 3, entry 7) and also reveals the potential of 2OG-competitive 2-oxo acids for selective 2OG oxygenase inhibition (the mode of $\mathrm{AspH}$ inhibition by 13 has previously been shown to be 2OG-competitive ${ }^{21}$ ).

Interestingly, varying the substitution pattern of the $2 \mathrm{OG}$ derivatives can result in a reversal of inhibition selectivity. For example, 3-ethyl 2OG (2) inhibits AspH more efficiently than FIH $\left(\mathrm{IC}_{50} \sim 1.2 \mu \mathrm{M}\right.$ for $\mathrm{AspH}$ and $\sim 4.6 \mu \mathrm{M}$ for $\mathrm{FIH}$; AspH:FIH inhibitor selectivity $\sim 4: 1$; Table 3 , entry 3 ), however, substituting its C3 ethyl with a propyl group reverses inhibition selectivity; the corresponding $2 \mathrm{OG}$ derivative 3 inhibits $\mathrm{FIH}$ more efficiently than $\mathrm{AspH}\left(\mathrm{IC}_{50} \sim\right.$ $5.7 \mu \mathrm{M}$ for $\mathrm{AspH}$ and $\sim 2.7 \mu \mathrm{M}$ for $\mathrm{FIH}$; AspH:FIH inhibitor selectivity $\sim 1: 2$; Table 3 , entry 4 ). Similarly, the substitution of a $2 \mathrm{OG} \mathrm{CH}_{2}$-unit by an $\mathrm{NH}$-unit can result in a reversal of inhibition: While 4-benzyl-2OG (19) is more efficient in inhibiting $\mathrm{AspH}$ compared to FIH (AspH:FIH inhibitor selectivity: 7:1; Table 3, entry 12), its NH-containing derivative NOFD is substantially more efficient in inhibiting FIH rather than AspH (AspH:FIH inhibitor selectivity: 1:50; Table 3, entry 1).

2OG derivative 28 neither inhibits $\mathrm{AspH}$ nor $\mathrm{FIH}$ catalysis (Table 3, entry 13) and also derivatives of $\mathbf{2 8}$ bearing substituents at the aromatic core do not inhibit the two oxygenases (Supplementary Table 1). In principle, selective 2OG oxygenase inhibition by $2 \mathrm{OG}$ derivatives bearing an aromatic or heteroaromatic core appears to be feasible, as revealed by the inhibition profile of $2 \mathrm{OG}$ derivative 35 , which bears a thiophene moiety and selectively inhibits AspH over FIH (Table 3, entry 14).

The inhibition results suggest that the ratio of $\mathrm{FIH}$ to $\mathrm{AspH}$ activity may be perturbed by using an AspH-selective inhibitor in the presence of the natural cosubstrate 2OG, a strategy that is complementary to the one previously investigated relying on the use of an FIH-selective cosubstrate (i.e., 14; Fig. 2f). AspH was most selectively inhibited by 4,4-dimethyl-2OG (13), which binds to AspH efficiently and is slowly converted into 2,2-dimethyl succinate (Table 3, entry 8$)^{21}$; therefore, FIH- and AspH-catalyzed substrate hydroxylations were simultaneously assessed in a single reaction vessel using SPE-MS in the presence of a physiological relevant 2OG concentration $(0.5 \mathrm{mM})$ and varied concentrations of 13 (Fig. 4). Increasing the concentration of $\mathbf{1 3}$ from 0 to $2.0 \mathrm{mM}$ reveals selective AspH inhibition in a dose-dependent manner, with no effect on $\mathrm{FIH}$ activity being observed. In the absence of the inhibitor 13, AspH 


\section{Table 3 Synthetic 20G derivatives inhibit FIH.}

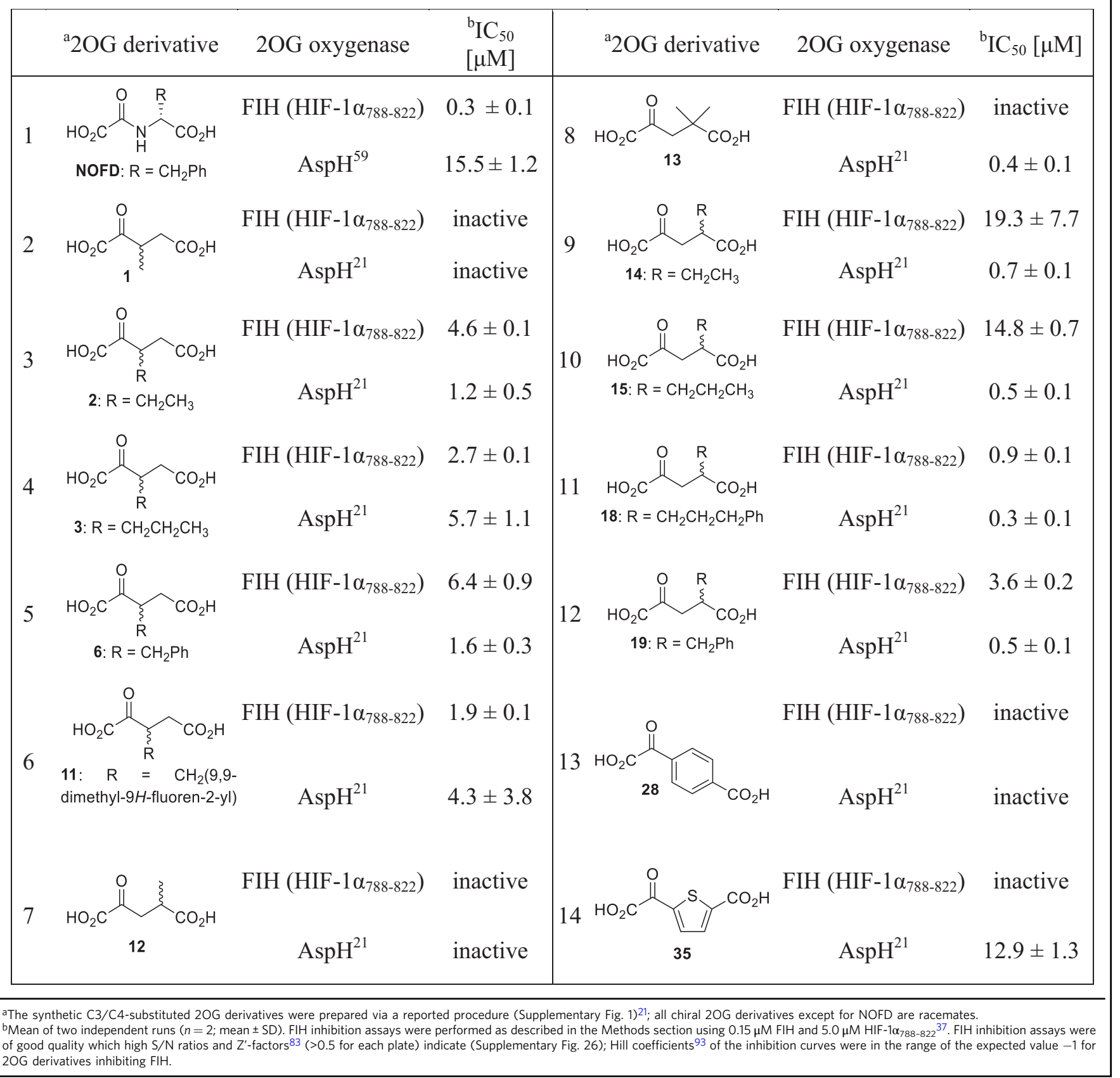

catalysis is substantially faster than $\mathrm{FIH}$ catalysis, with substrate hydroxylation reaching apparent completion after $\sim 1 \mathrm{~min} ; \sim 80 \%$ FIH-catalyzed substrate turnover was only observed after 4 min (Fig. 4a). Using equimolar amounts of $2 \mathrm{OG}$ and 13 (0.5 $\mathrm{mM}$ each), $\sim 80 \%$ conversion is observed for $\mathrm{FIH}$ after $4 \mathrm{~min}$ while only $\sim 60 \%$ conversion is observed for AspH (Fig. 4d). In the presence of a fourfold excess of 13 with respect to $2 \mathrm{OG},<20 \%$ of AspH-catalyzed substrate hydroxylation was observed after $4 \mathrm{~min}$ (Fig. 4f). A comparable result was obtained in a titration experiment with $2 \mathrm{OG}$ and 4-ethyl-2OG (14), which is an alternative FIH cosubstrate and an AspH inhibitor (Fig. 2f and Table 3, entry 9) ${ }^{21}$. The titration revealed that a tenfold excess of $\mathbf{1 4}$ over 2OG substantially slows down AspH catalysis while showing only a marginal effect on FIH catalysis
(Supplementary Fig. 27). The results reveal the potential of 2-oxo acids for selective 2OG oxygenase inhibition at physiologically relevant $2 \mathrm{OG}$ concentrations.

Mechanism of FIH inhibition. To investigate the mechanism by which the synthetic $2 \mathrm{OG}$ derivatives inhibit $\mathrm{FIH}$, the concentrationdependent effect of the potent FIH inhibitor 18 on the 2OG $K_{m}^{\mathrm{app}}$-value was investigated using HIF-1 $\alpha_{788-822}$ (Supplementary Fig. 28). The $2 \mathrm{OG} K_{m}^{\mathrm{app}}$-value decreases in a linear fashion with ascending inhibitor concentrations, while the $v_{\max }^{\mathrm{app}}$-values converge, indicating a linear 2OG-competitive inhibition mechanism (Fig. 5a). Using non-linear regression, the $K_{i}$-value of $\mathbf{1 8}$ was determined to 
a
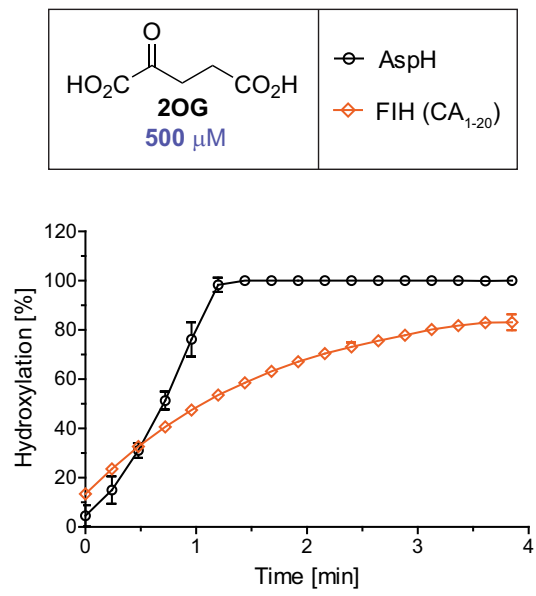

d
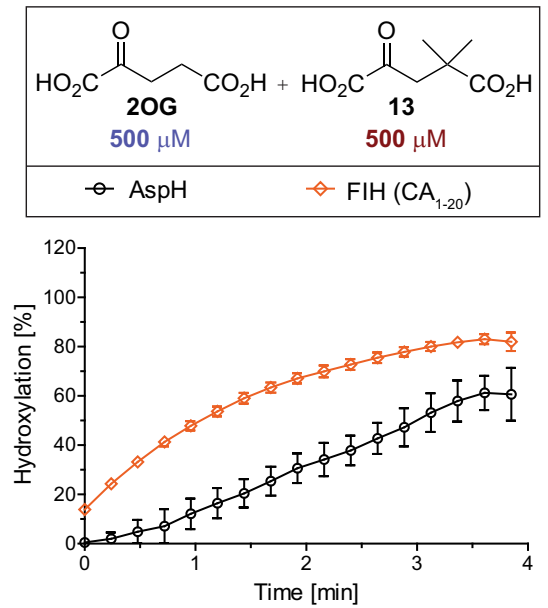

b
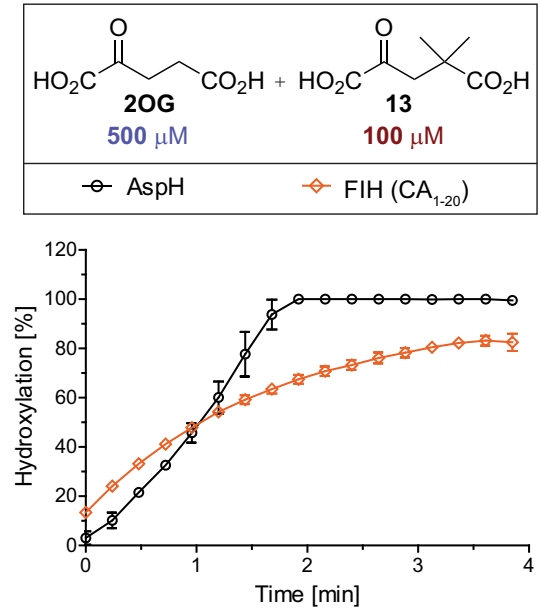

e
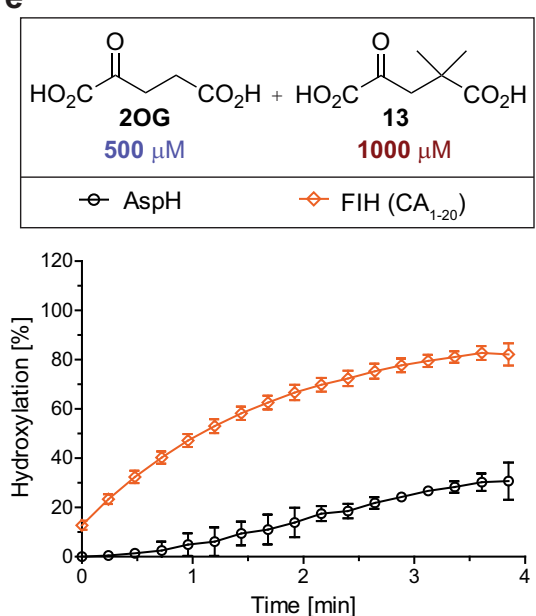

C
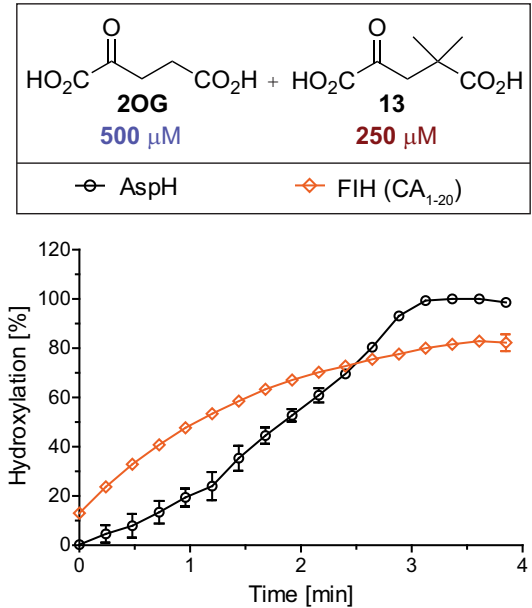

f
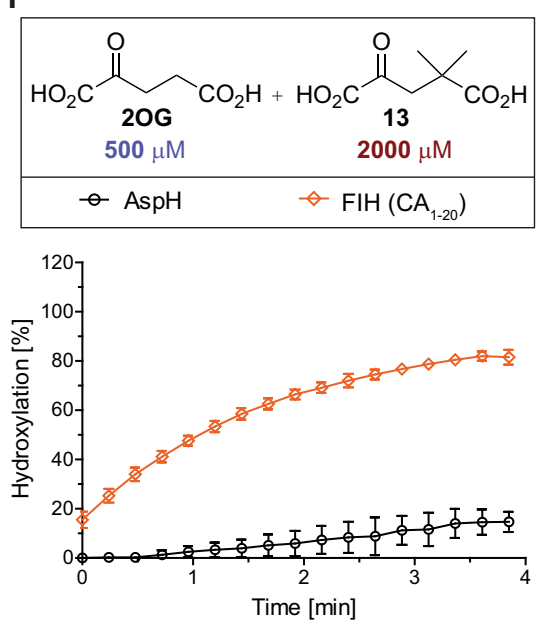

Fig. 4 4,4-Dimethyl-2OG selectively inhibits AspH in the presence of FIH at physiological $20 \mathrm{G}$ concentrations. $20 \mathrm{G}$ oxygenase assays were performed as described in the Methods section using $0.15 \mu \mathrm{M}$ of each $2 \mathrm{OG}$ oxygenase (diamond: $\mathrm{FIH}$, circle: $\mathrm{AspH}$ ) and $5.0 \mu \mathrm{M}$ of each substrate (orange: $\mathrm{CA}_{1-20}{ }^{47}$; black: hFX-EGFD1 ${ }_{86-124}-4 \mathrm{Ser}^{31}, \mathrm{AspH}$ substrate) in the presence of $100 \mu \mathrm{M}$ LAA, $10 \mu \mathrm{M} \mathrm{FAS}$, and the indicated concentrations of cosubstrates in buffer ( $50 \mathrm{mM}$ Tris, $50 \mathrm{mM} \mathrm{NaCl}, \mathrm{pH}$ 7.5). Mean of three independent runs $(n=3$; mean \pm SD). Measurement times were normalized to the first sample injection analyzed after the addition of $20 \mathrm{G}$ oxygenase to the Substrate Mixture ( $t=0 \mathrm{~min}$ ), by which time low levels of hydroxylation were manifest. Titration of 4,4-dimethyl-2OG (13) to a reaction mixture containing $500 \mu \mathrm{M} 20 \mathrm{G}$ : a $0 \mu \mathrm{M}$ 13; b $100 \mu \mathrm{M}$ 13; c $250 \mu \mathrm{M}$ 13; d $500 \mu \mathrm{M} 13 ; \mathbf{e} 1000 \mu \mathrm{M}$ 13; f $2000 \mu \mathrm{M} 13$. Source data are provided as a Source Data file.

be $\sim 0.2 \mu \mathrm{M}$. Analysis of a Cornish-Bowden $\operatorname{plot}^{63}$ further supports a 2OG-competitive inhibition mode, i.e., parallel lines for different $2 \mathrm{OG}$ concentrations are obtained when plotting [2OG] $/ v$ against [18] and their intercept with the $y$ axis increases on increasing the $2 \mathrm{OG}$ concentration (Fig. 5b).

FIH was crystallized in the presence of $\mathrm{Zn}$ (II) and the $2 \mathrm{OG}$ derivatives 11 or $\mathbf{1 8}$ to investigate their binding modes. FIH crystallized in the $P 4_{1} 22_{1} 2$ space group (FIH:11, $1.99 \AA$ resolution; FIH:18, $1.90 \AA$ resolution; Supplementary Figs. 29 and 31). Clear electron density corresponding to $\mathbf{1 8}$ or $\mathbf{1 1}$, respectively, was observed in the active site (Fig. $5 \mathrm{c}$ and d); 11 and $\mathbf{1 8}$ bind FIH in a similar manner as 2OG (Fig. 5e). The analysis of the electron density maps for the 2OG derivatives indicates that only $(S)-11$ and $(S)-18$ bind FIH, even though both were present as racemic mixtures (Supplementary Figs. 30 and 32).

The conformation of $(S)-\mathbf{1 8}$ in the FIH:18 structure is reminiscent of the NOFD conformation in the reported FIH:NOFD ${ }^{52}$ structure, indicating that these molecules inhibit FIH by a similar mechanism: their bulky phenyl substituents extent into the FIH substrate-binding pocket and appear to shield the active site metal from the substrate and/or $\mathrm{O}_{2}$ (Fig. 5e). By contrast, the rigid and relatively bulky fluorenyl substituent of $(S)-11$ orients in a different manner than the substituents of $(S)$ 18 and NOFD, triggering a conformational change in the Tyr102 $2_{\mathrm{FIH}}$ sidechain. In the FIH:11 structure, the Tyr102 $\mathrm{FIH}$ sidechain faces into the substrate-binding position, likely to avoid a steric clash with the fluorenyl substituent of $(S)-\mathbf{1 1}$, and thus blocks the substrate from binding to the FIH active site (red arrow in Fig. 5f). Movement of the Tyr $102_{\mathrm{FIH}}$ sidechain has been previously observed in crystal structures of FIH complexed with different substrates (Supplementary Fig. 33) ${ }^{43}$. In agreement with the proposal that the coordination of $(S)-\mathbf{1 1}$ and $(S)-\mathbf{1 8}$ to the FIH active site impairs substrate binding, FIH crystals with both inhibitor and substrate-bound could not be obtained. Even though inhibitors $\mathbf{1 1}$ and $\mathbf{1 8}$ display no substantial selectivity between $\mathrm{AspH}$ and $\mathrm{FIH}$, the crystallographic analyses may help in the design of selective FIH inhibitors.

\section{Discussion}

Different strategies can be envisaged in which $20 \mathrm{O}$ derivatives might be used to alter the normal reaction outcomes of one or 
a

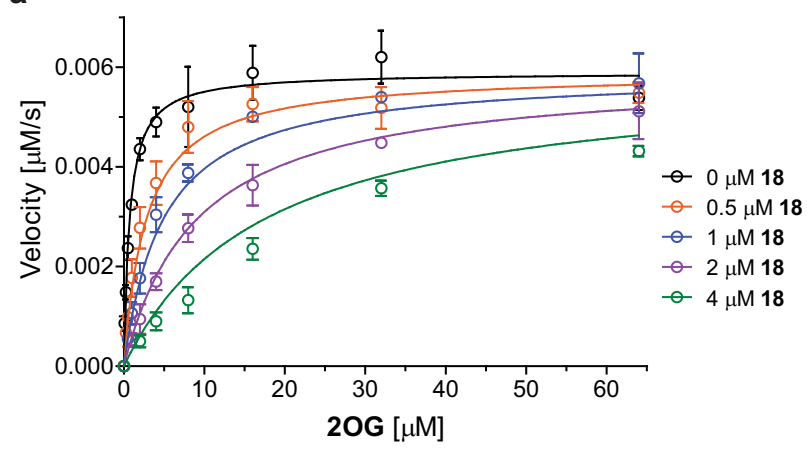

C

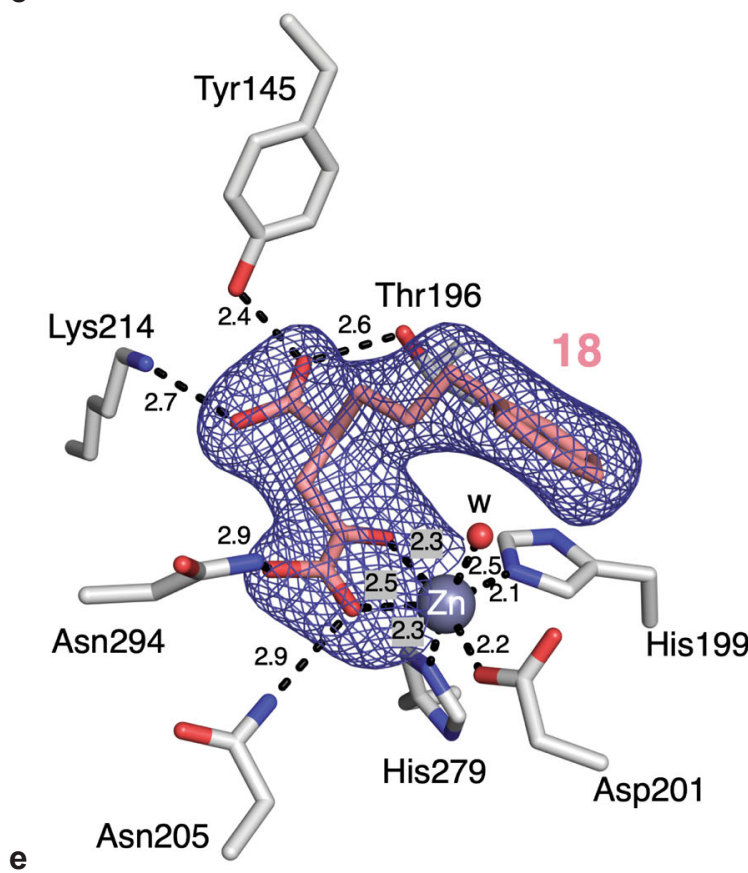

e

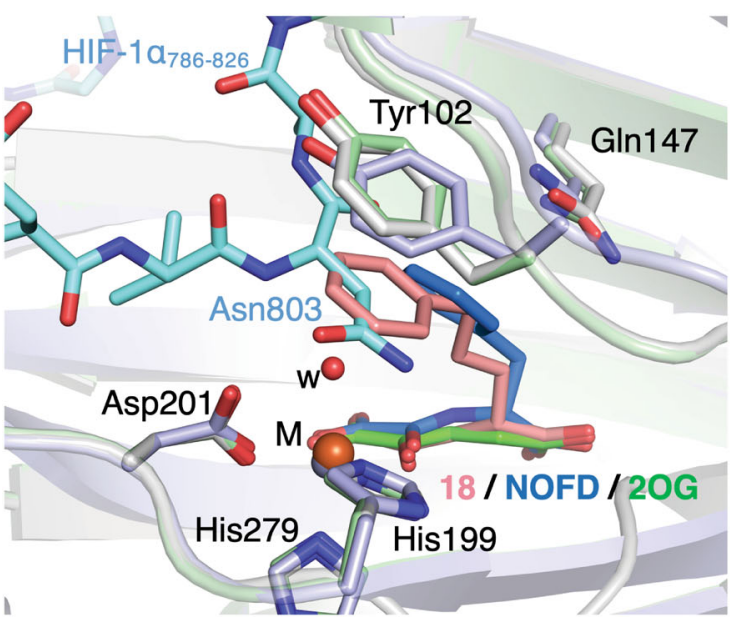

b

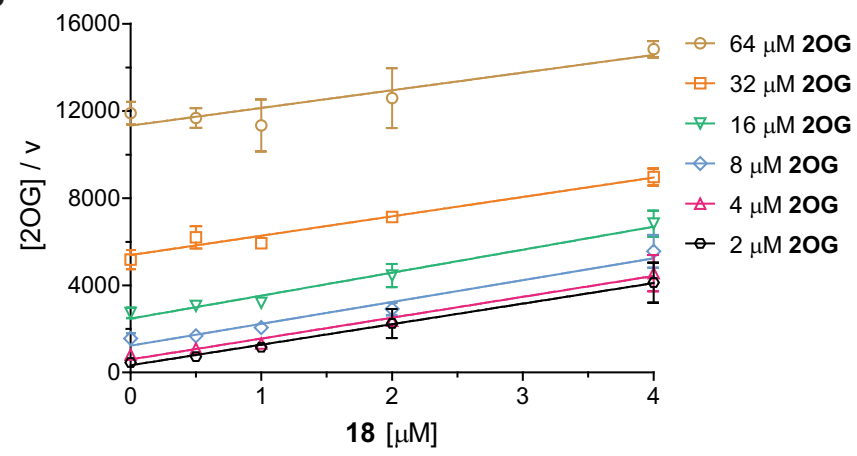

d

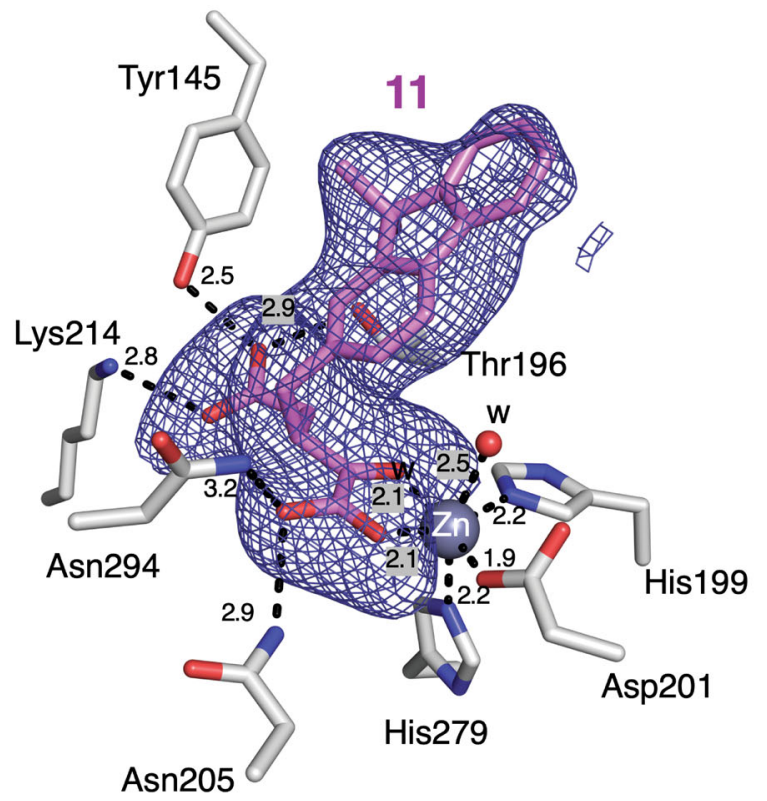

f

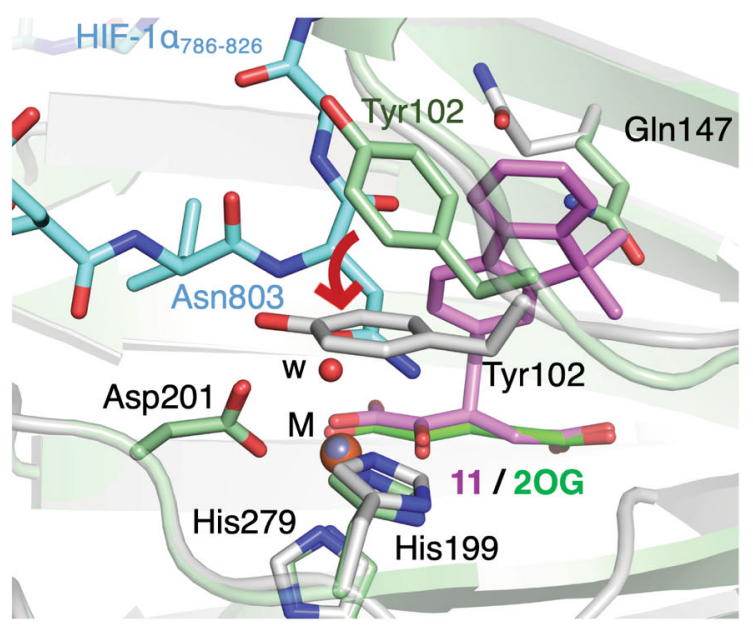

principle studies that $2 \mathrm{OG}$ derivatives can be used to enable strategy 1 (Fig. 6a), i.e., to selectively modulate the activity of one or both of two human 2OG oxygenases, which both catalyze the hydroxylation of Asn- and Asp-residues, i.e., FIH and AspH.

Our results of screens of $2 \mathrm{OG}$ derivatives with $\mathrm{FIH}$ and $\mathrm{AspH}$ led to the identification of two complementary approaches to selectively modulate the activities of FIH and AspH (strategy 1, Fig. 6a). FIH activity can be selectively enhanced in the presence of AspH using either an FIH-selective cosubstrate (i.e., 14; Fig. 2f) 
Fig. 5 The 20 G derivatives 11 and 18 inhibit FIH by different modes. a Effect of variable $\mathbf{1 8}$ concentrations on the Michaelis-Menten kinetics. Mean of three independent runs $\left(n=3\right.$; mean \pm SD); $\mathbf{b}$ Cornish-Bowden plot ${ }^{63}$ for $\mathbf{1 8}$; $\mathbf{c}$ and $\mathbf{d}$ representative OMIT electron density map $\left(\mathrm{mF}_{\mathrm{o}}-\mathrm{DF}_{\mathrm{c}}\right)$ contoured to c $5.0 \sigma$ around (S)-18 of the $\mathrm{FIH}: \mathbf{1 8}$ structure (FIH: gray; $\mathrm{Zn}$ ion: lavender blue; carbon-backbone of 18: salmon; w: water) and $\mathbf{d} 3.0 \sigma$ around (S)-11 of the $\mathrm{FIH}: \mathbf{1 1}$ structure (FIH: gray; Zn ion: lavender blue; carbon-backbone of 11: violet; w: water), respectively; e superimposition of a view from the FIH:11 structure with views from the reported FIH:NOFD (FIH: light blue; Fe ion: orange; carbon-backbone of NOFD: deep blue; PDB ID: 1YCI ${ }^{52}$ ) and FIH:2OG:HIF$1 \alpha_{786-826}$ (FIH: pale green; Fe ion: orange; carbon-backbone of 2OG: green; carbon-backbone of HIF-1 $\alpha_{786-826}$ : cyan; PDB ID: $1 \mathrm{H} 2 \mathrm{~L}^{61}$ ) structures reveals a similar mode of inhibition for NOFD and 18; $\mathbf{f}$ superimposition of a view from the FIH:11 structure with one from the reported FIH:2OG:HIF-1 $\alpha_{786-826}$ structure (FIH: pale green; Fe ion: orange; carbon-backbone of 2OG: green; carbon-backbone of HIF-1 $\alpha_{786-826}$ : cyan; PDB ID: $1 \mathrm{H} 2 \mathrm{~L}^{61}$ ) reveals that the binding of $\mathbf{1 1}$ to FIH triggers conformational changes in the Tyr102 $\mathrm{FIH}$ sidechain. Source data are provided as a Source Data file, information on crystallographic data is provided in the Data availability section.

a

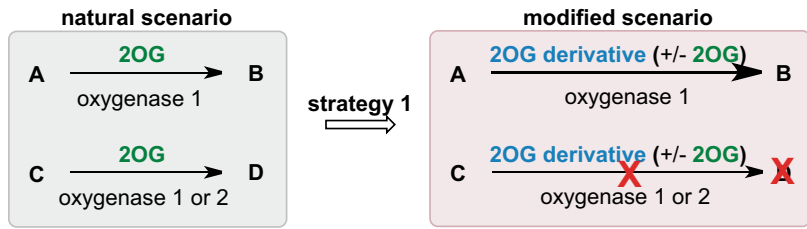

b

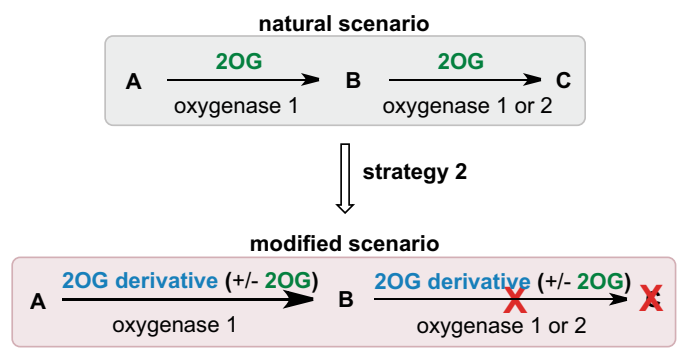

C

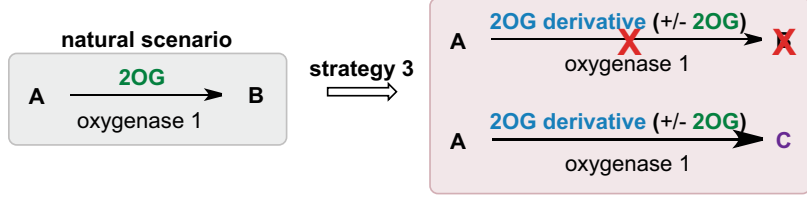

Fig. 620 derivatives may be used to selectively control reaction outcomes of $20 \mathrm{G}$ oxygenase catalysis. Each enzyme reaction involves two-electron substrate oxidation coupled to the conversion of $2 \mathrm{OG} / \mathrm{O}_{2}$ to succinate $/ \mathrm{CO}_{2}$. The substitution of $2 \mathrm{OG}$ by a $2 \mathrm{OG}$ derivative or the addition of an inhibitory $20 \mathrm{O}$ derivative has the potential to alter natural reaction outcomes of one or multiple 20G oxygenase reactions. For example, it may enable the selective enhancement/inhibition of catalysis by one $20 \mathrm{G}$ oxygenase in the presence of another/others (a strategy 1), the perturbation of sequential 2OG oxygenase reactions (b strategy 2), or the formation of different reaction products in a 2-oxo acid cosubstratedependent manner (c strategy 3).

in the absence of 2OG, or an AspH-selective inhibitor in the presence of a physiologically relevant concentration of $2 \mathrm{OG}$ (i.e., 13; Fig. 4). AspH activity can be selectively enhanced in the presence of FIH using a partially AspH-selective cosubstrate (i.e., 28; Supplementary Fig. 8) in the absence of 2OG. High levels of cosubstrate/inhibitor selectivity were observed in all cases, revealing the potential of 2-oxo acids for the selective enhancement and/or inhibition of $2 \mathrm{OG}$ oxygenase catalysis. This potential is supported by the reported lack of cosubstrate/inhibition activity of some of the tested C4-alkyl-substituted 2OG derivatives with wild-type KDM4A, which is a 2OG-dependent JmjC $\mathrm{KDM}^{19}$.

The application of MS-based assays to simultaneously assess $\mathrm{FIH}$ and AspH activity in the same reaction vessel complements kinetic studies with isolated 2OG oxygenases and enables the direct comparison of $2 \mathrm{OG}$ oxygenase activities. Importantly, the simultaneous turnover assays show that natural products can have a profound impact on the reactivity profiles of isolated $2 \mathrm{OG}$ oxygenases. For example, 3-methyl-2OG (1), which is an ingredient of honey ${ }^{48}$, promotes $\mathrm{AspH}$ catalysis relative to $\mathrm{FIH}$ catalysis, whereas its regioisomer, 4-methyl-2OG (12), which is present in plants 49,50 and in wine ${ }^{51}$, promotes FIH catalysis relative to $\mathrm{AspH}$ catalysis in the absence of $2 \mathrm{OG}$ (Fig. 2; strategy 1, Fig. 6a). Considering that cancer-associated mutations of the human isocitrate-dehydrogenase complex result in a $2 \mathrm{OG}$ deficient phenotype which, along with elevated 2-hydroxyglutarate levels, can correlate with decreased 2OG oxygenase activity ${ }^{64,65}$, the result that naturally occurring $20 \mathrm{OG}$ derivatives can enable $2 \mathrm{OG}$ oxygenase activity could be of in vivo relevance and medicinal value, e.g., to treat side effects arising from reduced $2 \mathrm{OG}$ oxygenase activity.

Some proteins, such as human Notch, are substrates of both AspH and FIH: AspH catalyzes the hydroxylation of extracellular Notch EGFDs ${ }^{66}$ and FIH catalyzes the hydroxylation of ankyrin repeats in the Notch intracellular domain ${ }^{39}$. Nature likely controls the FIH and AspH-catalyzed post-translational Notch modifications by spatial separation of the enzymes (FIH is predominantly present in the cytosol ${ }^{67}$, whereas the oxygenase domain of $\mathrm{AspH}$ faces into the $\mathrm{ER}^{68}$ ). The use of a selective cosubstrate may enable the selective enhancement of AspH or FIH catalysis acting on the same substrate, thus complementing natural control by spatial separation. Although there is likely scope for improvement of the synthetic $20 \mathrm{OG}$ derivatives reported by us, our studies provide proof-of-principle for this concept, i.e., 4-carboxyphenylglyoxylic acid (28) is a substantially more efficient AspH than FIH cosubstrate (Supplementary Fig. 8), whereas 4-ethyl-2OG (14) is an FIH cosubstrate but an AspH inhibitor (Fig. 2f).

The comparison of FIH:2OG derivative structures with reported AspH:2OG derivative structures reveals that the unusual $\mathrm{Fe}(\mathrm{II})$-binding geometry of $\mathrm{AspH}$, which has only two $\mathrm{Fe}$ (II) binding ligands compared to the typical triad present in other $2 \mathrm{OG}$ hydroxylases (including in FIH and KDM4A) ${ }^{21}$, does not appear to underpin the observed differences in cosubstrate selectivity patterns. In near all the FIH:2OG derivative and AspH:2OG derivative structures, the $2 \mathrm{OG}$ derivatives occupy the 2OG-binding site and display similar conformations as $2 \mathrm{OG}$ (Fig. 3); a notable exception is the structurally distinct 2OG derivative 22, whose conformation differs owing to its cyclic structure (Fig. 3e). Together with the reported lack of cosubstrate activity of certain C4-substituted 2OG derivatives with KDM4 $\mathrm{A}^{19}$, these observations suggest that the interactions of the C3/C4-substituents of the 2OG derivatives with sidechains of (hydrophobic) residues flanking the 2OG-binding site, which form hydrophobic regions, determine the efficiency of cosubstrate binding and coproduct release (Fig. 3f). In general, the FIH $k_{\text {cat }} / K_{m}$-values for the $2 \mathrm{OG}$ derivatives appear to decrease upon increasing the length of the $2 \mathrm{OG}$ C4 substituent from hydrogen to 
methyl and ethyl, independent from the nature of the substrate (Table 2). As the $K_{m}^{\text {app }}$-values remain in the same range within experimental error $(0.5 \sim 3.1 \mu \mathrm{M}$, Table 2$)$, this observation indicates that the 2OG derivatives bind FIH with similar affinities, but likely undergo subsequent oxidative decarboxylation reactions less efficiently than 2OG, resulting in apparent inhibition. Although they employ the same general strategies for $2 \mathrm{OG}$ binding $^{69}$, the 2OG-binding sites of different $2 \mathrm{OG}$ oxygenases vary substantially and thus may be particularly suited for the binding of specific $2 \mathrm{OG}$ derivatives resulting either in selective inhibition or activation as evidenced by our work.

The results presented here highlight the potential of 2-oxo acids, including $2 \mathrm{OG}$ derivatives and natural products, to inhibit $2 \mathrm{OG}$ oxygenases and related enzymes. The potential of 2-oxo acids for the inhibition of 2OG oxygenases is supported by the (partially) selective FIH inhibitor NOFD, a derivative of the broader spectrum $2 \mathrm{OG}$ oxygenase inhibitor $\mathrm{NOG}^{52}$. The inhibition selectivity of NOG derivatives has also been studied for other subclasses of $2 \mathrm{OG}$ oxygenases including the JmjC KDMs and TET enzymes ${ }^{70-73}$. The observation that dimethyl $N$-oxalylglycine (DMOG), a cell-wall penetrable NOG precursor, inhibits $2 \mathrm{OG}$ oxygenases in vivo and in cellular studies, supports the potential utility of corresponding $2 \mathrm{OG}$ derivatives for cellular functional assignment studies, regardless of the high $2 \mathrm{OG}$ concentrations in cells $\left(\sim 1 \mathrm{mM} 2 \mathrm{OG}{ }^{57,58}\right)$. The potential of 2 -oxo acids to inhibit $2 \mathrm{OG}$ oxygenases is shown by the results for 4,4dimethyl-2OG (13), which efficiently and selectively inhibits AspH $\left(\mathrm{IC}_{50} \sim 0.4 \mu \mathrm{M}^{21}\right)$, even in the presence of physiologically relevant $2 \mathrm{OG}$ concentrations, i.e., $0.5 \mathrm{mM} 2 \mathrm{OG}$ (Fig. 4).

The involvement of 2-oxo acids in multiple metabolic pathways may be considered to limit the utility of 2OG derivatives for their development as human therapeutics. Indeed, the presence of 2OG derivatives with substituents at the $\mathrm{C} 3 / \mathrm{C} 4$ position may modulate the activities of 2OG-dependent enzymes other than $2 \mathrm{OG}$ oxygenases. For example, it has been reported that 4,4dimethyl-2OG (13) is a substantially less-efficient substrate of glutamic oxaloacetic aminotransferase compared with the natural substrate $2 \mathrm{OG}$ or 4 -methyl-2OG (12), suggesting that $\mathbf{1 3}$ might not interfere with the catalytic activities of human aminotransferases ${ }^{74}$. However, from a safety perspective, it is notable that some of the $2 \mathrm{OG}$ derivatives described here are present in human nutrition ${ }^{48-51}$ and cells appear to tolerate reasonably high levels of DMOG with no obvious toxicity ${ }^{75}$. Further, considering the pleiotropic effects that NOG and its derivatives exhibit in cellular studies, application of related 2-oxo acid derivatives to cells may trigger phenotypes useful in identifying previously overlooked 2OG oxygenase/2OG-utilizing enzyme functions. $\mathrm{FIH}^{76}$ and $\mathrm{AspH}^{22-26}$ are proposed medicinal chemistry targets and the use of (partially) selective 2OG derivatives, such as $\mathbf{1 3}$ or corresponding esters in cellular studies may help to improve the currently limited understanding on their biochemical roles and complement similar approaches using heterocyclic small-molecules ${ }^{24,76-81}$.

In summary, the combined results with FIH and AspH, $2 \mathrm{OG}$ oxygenases that both catalyze Asp- and Asn-residue hydroxylations, demonstrate the potential of 2-oxo acid derivatives to selectively enhance or inhibit one of the enzyme reactions in the presence of the other, in some cases in the presence of 2OG. From a set of $352 \mathrm{OG}$ derivatives, we identified 10 as enhancing and 17 as inhibiting FIH activity. Although further mechanistic studies are required to analyze the details of why some 2-oxo acid derivatives inhibit and others enable 2OG oxygenase catalysis, our structural studies provide insight into the active site regions into which the $\mathrm{C} 3 / \mathrm{C} 42 \mathrm{OG}$ substituents bind; this information should be useful in the identification of 2-oxo acid derivatives, including natural products present in food, that enable selective modulation of $2 \mathrm{OG}$ oxygenase reaction outcomes in vivo. Analogous strategies described here for modulation of $2 \mathrm{OG}$ oxygenase activity by cosubstrate derivatives should be applicable to other enzyme families employing modified cosubstrates.

\section{Methods}

Protein production and purification. Wildtype $\mathrm{N}$-terminally $\mathrm{His}_{6}$-tagged human FIH was produced and purified by an adaption of a literature procedure ${ }^{82}$. In brief, DNA encoding for wild-type human FIH with a N-terminal $\mathrm{His}_{6}$-tag was cloned into a pNIC28-Bsa4 plasmid. After transformation of the plasmid into E. coli BL21 (DE3) cells, expression was performed at $37^{\circ} \mathrm{C}$ in $\mathrm{LB}$ media supplemented with $37.5 \mu \mathrm{g} / \mathrm{mL}$ kanamycin. FIH-production was induced at an $\mathrm{OD}_{600}$ of $\sim 0.6$ at $20^{\circ} \mathrm{C}$ by adding isopropyl $\beta$-D-thiogalactopyranoside (final concentration of $0.5 \mathrm{mM}$ ). Cultures were grown overnight. Cells were spun at $5000 \mathrm{rpm}$ for $10 \mathrm{mins}$ and the resulting cell pellets were stored at $-80^{\circ} \mathrm{C}$

The cell pellets were suspended in buffer ( $50 \mathrm{mM}$ HEPES, pH 7.4, $500 \mathrm{mM}$ $\mathrm{NaCl}, 20 \mathrm{mM}$ imidazole, $0.5 \mathrm{mM}$ TCEP) and lysed by high-pressure homogenization (three passages). Following cell lysis, the insoluble cell debris was removed by centrifugation $\left(36,000 \times g, 1 \mathrm{~h}, 4^{\circ} \mathrm{C}\right)$. $\mathrm{His}_{6}$-tagged $\mathrm{FIH}$ was purified by sequential $\mathrm{Ni}$ (II)-affinity chromatography (using 50 mM HEPES, pH 7.4, $1 \mathrm{M}$ $\mathrm{NaCl}, 0.5 \mathrm{mM}$ TCEP with increasing imidazole concentrations: $20-300 \mathrm{mM}$ ) and size-exclusion chromatography (HiLoad 16/600 Superdex 75 pg column; buffer: $20 \mathrm{mM}$ HEPES, pH 7.4, $500 \mathrm{mM} \mathrm{NaCl}, 5 \%$ glycerol, $0.5 \mathrm{mM}$ TCEP) using an ÄKTAxpress machine. Fractions containing FIH were pooled and concentrated; FIH was $>95 \%$ pure by SDS-PAGE and MS analysis. Purified FIH was stored at a concentration of $42 \mu \mathrm{M}(20 \mathrm{mM}$ HEPES, pH 7.4, $500 \mathrm{mM} \mathrm{NaCl}, 5 \%$ glycerol, $0.5 \mathrm{mM}$ TCEP) at $-78^{\circ} \mathrm{C}$, fresh aliquots were used for all FIH assays (cleavage of the $\mathrm{His}_{6}$-tag did not show beneficial effects on FIH activity).

A truncated construct of wild-type $\mathrm{N}$-terminally $\mathrm{His}_{6}$-tagged human $\mathrm{AspH}$, comprising the catalytic oxygenase domain and the tetratricopeptide repeat domain $\left(\mathrm{His}_{6}-\mathrm{AspH}_{315-758}\right)$, was produced and purified as previously reported ${ }^{27,31}$.

Substrate peptides. FIH substrates were based on the sequence of the two reported human FIH substrate proteins HIF-1 $\alpha^{34,36}$ (HIF-1a C-terminal transactivation domain fragment amino acids 788-822, HIF-1 $\alpha_{788-822}$ : DESGLPQLTSYDCEVNAPIQGSRNLLQGEELLRAL; FIH catalyzes hydroxylation of Asn $803^{34,37}$ ) and tankyrase- ${ }^{62}$ (tankyrase-2 amino acids 691-710, TNKS2 ${ }_{691-710}$ : NLEVAEYLLQHGADVNAQDK; FIH catalyzes hydroxylation of Asn706 ${ }^{62}$ ) and of a reported synthetic consensus ankyrin repeat ${ }^{46}\left(\mathrm{CA}_{1-20}:{ }^{47}\right.$ HLEVVKLLLEAGADVNAQDK; FIH catalyzes hydroxylation of Asn16 ${ }^{47}$ ). The AspH substrate peptide, i.e., hFX-EGFD1 $1_{86-124}-4 \mathrm{Ser}^{27,31}$, was based on the sequence of the EGFD1 (amino acids 86-124) of the reported AspH substrate human coagulation factor X (hFX), four hFX cysteine residues (Cys $90_{\mathrm{hFX}}$, Cys $95_{\mathrm{hFX}}$, Cys $112_{\mathrm{hFX}}, \mathrm{Cys} 121_{\mathrm{hFX}}$ ) have been substituted for serine residues to avoid disulfide scrambling. Peptides were synthesized by solid-phase peptide synthesis and purified by GL Biochem (Shanghai) Ltd (Shanghai, China); all peptides were prepared with C-terminal amides.

FIH inhibition assays. FIH inhibition assays were performed as described ${ }^{44}$, using cosubstrate/cofactor stock solutions (L-ascorbic acid, LAA: $50 \mathrm{mM}$ in MQ-grade water; 2-oxoglutarate, 2OG: $10 \mathrm{mM}$ in MQ-grade water; ammonium iron(II) sulfate hexahydrate, FAS, $\left(\mathrm{NH}_{4}\right)_{2} \mathrm{Fe}\left(\mathrm{SO}_{4}\right)_{2} \cdot 6 \mathrm{H}_{2} \mathrm{O}: 400 \mathrm{mM}$ in $20 \mathrm{mM} \mathrm{HCl}$ diluted to $1 \mathrm{mM}$ in MQ-grade water), which were freshly prepared from commerciallysourced solids (Sigma Aldrich).

Dimethyl sulfoxide (DMSO) solutions of the 2OG derivatives were dry dispensed across 384-well polypropylene assay plates (Greiner) in an approximately threefold and 11-point dilution series $(100 \mu \mathrm{M}$ top concentration; the final DMSO assay concentration was kept constant at $0.5 \%_{\mathrm{v} / \mathrm{v}}$ ) using an ECHO 550 acoustic dispenser (Labcyte). DMSO and NOFD ${ }^{52}$ were used as negative and positive inhibition controls, respectively. Each reaction was performed in technical duplicates in adjacent wells of the assay plates; additionally, assays were performed in two independent duplicates on different days using different inhibitor solutions.

The Enzyme Mixture $\left(25 \mu \mathrm{L} /\right.$ well), containing $0.3 \mu \mathrm{M} \mathrm{His}_{6}$-FIH in buffer (50 mM Tris, $50 \mathrm{mM} \mathrm{NaCl}, \mathrm{pH} 7.5$ ), was dispensed across the inhibitor-containing 384-well assay plates with a multidrop dispenser (ThermoFischer Scientific) at $20^{\circ} \mathrm{C}$ under an ambient atmosphere. The plates were subsequently centrifuged (1000 rpm, $15 \mathrm{~s})$ and incubated for $15 \mathrm{~min}$ at $20^{\circ} \mathrm{C}$. The Substrate Mixture $(25 \mu \mathrm{L} /$ well), containing $10.0 \mu \mathrm{M}$ HIF- $1 \alpha_{788-822}{ }^{37}$ substrate, $200 \mu \mathrm{M}$ LAA, $20.0 \mu \mathrm{M} 2 \mathrm{OG}$, and $20.0 \mu \mathrm{M}$ FAS in buffer $(50 \mathrm{mM}$ Tris, $50 \mathrm{mM} \mathrm{NaCl}, \mathrm{pH} 7.5)$, was added using the multidrop dispenser. The plates were centrifuged $(1000 \mathrm{rpm}, 15 \mathrm{~s})$ and after incubating for $15 \mathrm{~min}$, the enzyme reaction was stopped by the addition of $10 \% \mathrm{v} / \mathrm{v}$ aqueous formic acid ( $5 \mu \mathrm{L} /$ well). The plates were then centrifuged (1000 rpm, $30 \mathrm{~s}$ ) and analyzed by MS.

MS-analyses were performed using a RapidFire RF 365 high-throughput sampling robot (Agilent) attached to an iFunnel Agilent 6550 accurate-mass quadrupole time-of-flight mass spectrometer operated in the positive ionization mode. Assay samples were aspirated under vacuum for $0.6 \mathrm{~s}$ and loaded onto a C4 SPE cartridge. After loading, the C4 SPE cartridge was washed with $0.1 \% \mathrm{v} / \mathrm{v}$ aqueous formic acid to remove non-volatile buffer salts $(5.5 \mathrm{~s}, 1.5 \mathrm{~mL} / \mathrm{min})$. The 
peptide was eluted from the SPE cartridge with $0.1 \%_{\mathrm{v} / \mathrm{v}}$ aqueous formic acid in $85 /$ $15 \mathrm{v} / \mathrm{v}$ acetonitrile/water into the mass spectrometer $(5.5 \mathrm{~s}, 1.25 \mathrm{~mL} / \mathrm{min})$ and the SPE cartridge re-equilibrated with $0.1 \%_{\mathrm{v} / \mathrm{v}}$ aqueous formic acid $(0.5 \mathrm{~s}, 1.25 \mathrm{~mL} /$ $\mathrm{min})$. The mass spectrometer was operated using the MassHunter Workstation B.08.00 software (Agilent), the mass spectrometer parameters were: capillary voltage $(4000 \mathrm{~V})$, nozzle voltage $(1000 \mathrm{~V})$, fragmentor voltage $(365 \mathrm{~V})$, gas temperature $\left(280^{\circ} \mathrm{C}\right)$, gas flow $(13 \mathrm{~L} / \mathrm{min})$, sheath gas temperature $\left(3500^{\circ} \mathrm{C}\right)$, sheath gas flow $\left(12 \mathrm{~L} / \mathrm{min}\right.$ ). The $\mathrm{m} / \mathrm{z}+2$ (for $\mathrm{CA}_{1-20}$ ) or +3 (for HIF-1 $\alpha_{788-822}$ ) charge states of the peptide (substrate) and the hydroxylated peptide (product) were used to extract ion chromatogram data, peak areas were integrated using RapidFire Integrator 4.3.0 (Agilent). Data were exported into Microsoft Excel and used to calculate the \% conversion of the hydroxylation reaction using the equation: \% conversion $=100 \times($ integral product peptide $) /($ integral substrate peptide + integral product peptide). Normalized dose-response curves (NOFD and DMSO controls) were obtained from the raw data by non-linear regression (GraphPad Prism 5) and used to determine $\mathrm{IC}_{50}$ values. The standard deviation (SD) of two independent $\mathrm{IC}_{50}$ determinations $(n=2)$ was calculated using GraphPad Prism 5. Z'-factors were calculated according to the cited literature using Microsoft Excel (Supplementary Fig. 26) ${ }^{83}$

Determination of kinetic parameters. Maximum velocities $\left(v_{\max }^{\mathrm{app}}\right)$ and Michaelis constants $\left(K_{m}^{\text {app }}\right)$ of FIH were determined in independent triplicates for $2 \mathrm{OG}$ and the synthetic 2 OG derivatives by SPE-MS monitoring HIF- $1 a_{788-822} 37$ or $\mathrm{CA}_{1-20} 47$ turnover. $\mathrm{His}_{6}-\mathrm{FIH}(1.8 \mu \mathrm{L}, 42 \mu \mathrm{M})$ was added at $20^{\circ} \mathrm{C}$ to a substrate mixture containing $5.0 \mu \mathrm{M}$ substrate, $100 \mu \mathrm{M}$ LAA, $20 \mu \mathrm{M}$ FAS, and 2OG/2OG derivative in $0.5 \mathrm{~mL}$ buffer ( $50 \mathrm{mM}$ Tris, $50 \mathrm{mM} \mathrm{NaCl}, \mathrm{pH}$ 7.5). Final 2OG/2OG derivative concentrations are given in Supplementary Figs. 5 and 6. Reactions were monitored with a rate of $\sim 1$ sample/40 $\mathrm{s}$ using the same SPE-MS configuration as described above. Data were analyzed as described above and the slopes of the initial reaction rates (Supplementary Figs. 5 and 6) fitted to a Michaelis-Menten plot using nonlinear regression (GraphPad Prism 5). The total concentration of active FIH was determined by an active site titration (Supplementary Fig. 7) and used to calculate turnover numbers $\left(k_{\mathrm{cat}}^{\mathrm{app}}\right)$ and specificity constants $\left(k_{\mathrm{cat}} / K_{m}\right)$ for $2 \mathrm{OG}$ and the $2 \mathrm{OG}$ derivatives.

Competition assays. To a Substrate Mixture containing $5.0 \mu \mathrm{M}$ of each of the requisite substrates (for FIH: HIF- $1 \alpha_{788-822}{ }^{37}$ and/or $\mathrm{CA}_{1-20}{ }^{47}$; for AspH: hFXEGFD1 $\left._{86-124}-4 \mathrm{Ser}^{27,31}\right), 100 \mu \mathrm{M}$ LAA, $10 \mu \mathrm{M}$ FAS, and $20 \mu \mathrm{M}$ 2OG/2OG derivative in $1.0 \mathrm{~mL}$ buffer $\left(50 \mathrm{mM}\right.$ Tris, $50 \mathrm{mM} \mathrm{NaCl}, \mathrm{pH}$ 7.5) was added $0.15 \mu \mathrm{M}$ of $\mathrm{His}_{6^{-}}$ $\mathrm{FIH}$ and $\mathrm{His}_{6}-\mathrm{AspH}_{315-758}$ (when appropriate). Peptide turnover was monitored by SPE-MS using the same instrument configuration (with the exception that the peptides were eluted from the SPE cartridge with $0.1 \%_{\mathrm{v} / \mathrm{v}}$ aqueous formic acid in $80 / 20_{\mathrm{v} / \mathrm{v}}$ acetonitrile/water) as described above.

Crystallography. Crystallization experiments were performed in 96-well, threesubwell, low profile Intelliplates (Art Robbins Instruments) using a Phoenix RE liquid dispensing robot (Art Robbins Instruments) with $1.6 \mathrm{M}$ ammonium sulfate, $6 \%_{\mathrm{w} / \mathrm{v}}$ PEG400, and 0.1 M HEPES buffer (pH 7.5) as the mother liquid. N-Terminally $\mathrm{His}_{6}$-tagged FIH $(20 \mathrm{mg} / \mathrm{mL})$ was mixed with $\mathrm{ZnCl}_{2}(1 \mathrm{mM}), 2 \mathrm{OG}$ derivative $(2 \mathrm{mM})$, and, when appropriate, a synthetic consensus ankyrin repeat peptide $\left(5 \mathrm{mM} ; \mathrm{CA}_{1-20}{ }^{47}\right)$ or a tankyrase- 2 fragment peptide ${ }^{62}(5 \mathrm{mM}$;

TNKS2 ${ }_{691-710}$ ) as FIH substrate. Crystals were grown using the vapor diffusion method at $20^{\circ} \mathrm{C}$ in $200 \mathrm{~nL}$ of $300 \mathrm{~nL}$ sitting drops with 2:1, 1:1, or 1:2 sample:well solution ratios; precipitants are listed in Supplementary Tables 2 and 3. Crystals were cryo-protected using mother liquor supplemented with $25 \%_{\mathrm{v} / \mathrm{v}}$ glycerol before cryo-cooling in liquid $\mathrm{N}_{2}$. Data were collected at $100 \mathrm{~K}$ using synchrotron radiation at diamond light source beamlines I03 and I04. Data were indexed, integrated, and scaled using the autoPROC ${ }^{84}$, STARANISO ${ }^{85}$, or $\mathrm{Xia}^{26}$ strategy of the beamline auto-processing pipeline (Supplementary Tables 2 and 3).

The FIH crystal structures were determined by MR using the AutoMR (PHASER ${ }^{87}$ ) subroutine in PHENIX (version 1.18.2) ${ }^{88}$. The search model used for MR was based on PDB ID $1 \mathrm{H} 2 \mathrm{~K}^{61}$ for FIH crystal structures. The structural model was improved by iterative cycles of manual re-building in Coot (version 0.8.6.1) ${ }^{89}$ and crystallographic refinement in phenix.refine ${ }^{90}$ (refinement details are summarized in Supplementary Tables 2 and 3). The crystal structure data for FIH complexed to Zn, 2OG derivative, and, in some cases, substrate (i.e. $\mathrm{CA}_{1-20}$ or TANK2 ${ }_{691-710}$ ) have been deposited in the protein data bank (see Data availability section). PyMOL (version 4.6) ${ }^{91}$ was used for the generation of graphical representations; polder omit maps were calculated using Polder Maps ${ }^{92}$ in PHENIX (version 1.18.2) ${ }^{88}$.

Reporting summary. Further information on research design is available in the Nature Research Reporting Summary linked to this article.

\section{Data availability}

The crystal structure data for FIH complexed to $\mathrm{Zn}, 2 \mathrm{OG}$ derivative (3-methyl-2OG, 1; 3-propyl-2OG, 3; 3-(9,9-dimethyl-9H-fluoren-2-yl)methyl-2OG, 11; 4-ethyl-2OG, 14; 4propyl-2OG, 15; 4-(3-phenylpropyl)-2OG, 18; 3-(carboxycarbonyl)cyclopentane-1carboxylic acid, 22), and, in some cases, substrate (i.e. $\mathrm{CA}_{1-20}$ or TANK2 ${ }_{691-710}$ ) have been deposited in the protein data bank under PDB accession codes: 7A1L (FIH:1), 7A1M (FIH:3), 7AlJ (FIH:18), 7A1K (FIH:11), 7A1N (FIH:1:CA $1-20)$, 7A1O (FIH:14:CA $\mathrm{CA}_{1-20}$ ), 7A1P (FIH:15:CA $\mathrm{CA}_{1-20}$ ), 7A1Q (FIH:22:CA $\mathrm{CA}_{1-20}$ ), and 7A1S (FIH:1:TANK2 $691-710$ ). In addition, data of the following reported crystal structure have been used: $1 \mathrm{H} 2 \mathrm{~L}^{61}, 1 \mathrm{H} 2 \mathrm{~K}^{61}, 1 \mathrm{YCI}^{52}$, and $6 \mathrm{YYX}^{21}$. Source data are provided with this paper.

Received: 2 June 2021; Accepted: 12 October 2021; Published online: 10 November 2021

\section{References}

1. 2-Oxoglutarate-Dependent Oxygenases (eds Hausinger, R. P. \& Schofield, C. J.). The Royal Society of Chemistry (2015).

2. Koivunen, P. et al. Inhibition of hypoxia-inducible factor (HIF) hydroxylases by citric acid cycle intermediates: possible links between cell metabolism and stabilization of HIF. J. Biol. Chem. 282, 4524-4532 (2007).

3. Laukka, T., Myllykoski, M., Looper, R. E. \& Koivunen, P. Cancer-associated 2 oxoglutarate analogues modify histone methylation by inhibiting histone lysine demethylases. J. Mol. Biol. 430, 3081-3092 (2018).

4. Tarhonskaya, H. et al. Studies on the interaction of the histone demethylase KDM5B with tricarboxylic acid cycle intermediates. J. Mol. Biol. 429, 2895-2906 (2017).

5. Laukka, T. et al. Fumarate and succinate regulate expression of hypoxiainducible genes via TET enzymes. J. Biol. Chem. 291, 4256-4265 (2016).

6. Raimundo, N., Baysal, B. E. \& Shadel, G. S. Revisiting the TCA cycle: signaling to tumor formation. Trends Mol. Med. 17, 641-649 (2011).

7. Losman, J.-A., Koivunen, P. \& Kaelin, W. G. 2-Oxoglutarate-dependent dioxygenases in cancer. Nat. Rev. Cancer 20, 710-726 (2020).

8. Sullivan, L. B., Gui, D. Y. \& Heiden, M. G. V. Altered metabolite levels in cancer: implications for tumour biology and cancer therapy. Nat. Rev. Cancer 16, 680-693 (2016)

9. Chowdhury, R. et al. The oncometabolite 2-hydroxyglutarate inhibits histone lysine demethylases. EMBO Rep. 12, 463-469 (2011).

10. Majamaa, K., Hanauske-Abel, H. M., Günzler, V. \& Kivirikko, K. I. The 2-oxoglutarate binding site of prolyl 4-hydroxylase. Eur. J. Biochem. 138, 239-245 (1984).

11. Cunliffe, C. J., Franklin, T. J., Hales, N. J. \& Hill, G. B. Novel inhibitors of prolyl 4-hydroxylase. 3. Inhibition by the substrate analog N-oxaloglycine and its derivatives. J. Med. Chem. 35, 2652-2658 (1992).

12. Martinez, S. \& Hausinger, R. P. Biochemical and spectroscopic characterization of the non-heme Fe(II)- and 2-oxoglutarate-dependent ethylene-forming enzyme from pseudomonas syringae pv. phaseolicola PK2. Biochemistry 55, 5989-5999 (2016).

13. Martinez, S. et al. Structures and mechanisms of the non-heme Fe(II)- and 2oxoglutarate-dependent ethylene-forming enzyme: substrate binding creates a twist. J. Am. Chem. Soc. 139, 11980-11988 (2017).

14. Mooney, B. P., Miernyk, J. A. \& Randall, D. D. The complex fate of $\alpha-$ ketoacids. Annu. Rev. Plant Biol. 53, 357-375 (2002).

15. Penteado, F. et al. a-Keto acids: acylating agents in organic synthesis. Chem. Rev. 119, 7113-7278 (2019).

16. Rohrbacher, F., Wucherpfennig, T. G. \& Bode, J. W. Chemical protein synthesis with the KAHA ligation. In: Protein Ligation and Total Synthesis II (ed Liu, L.). Springer International Publishing (2015).

17. Luo, Z., Yu, S., Zeng, W. \& Zhou, J. Comparative analysis of the chemical and biochemical synthesis of keto acids. Biotechnol. Adv. 47, 107706 (2021).

18. Cooper, A. J. L., Ginos, J. Z. \& Meister, A. Synthesis and properties of the aketo acids. Chem. Rev. 83, 321-358 (1983).

19. Breski, M., Dey, D., Obringer, S., Sudhamalla, B. \& Islam, K. Engineering biological $\mathrm{C}-\mathrm{H}$ functionalization leads to allele-specific regulation of histone demethylases. J. Am. Chem. Soc. 138, 13505-13508 (2016)

20. Mukherji, M. et al. 'Chemical co-substrate rescue' of phytanoyl-CoA 2hydroxylase mutants causing Refsum’s Disease. Chem. Commun. 972-973 (2001).

21. Brewitz, L., Nakashima, Y. \& Schofield, C. J. Synthesis of 2-oxoglutarate derivatives and their evaluation as cosubstrates and inhibitors of human aspartate/asparagine- $\beta$-hydroxylase. Chem. Sci. 12, 1327-1342 (2021).

22. Lavaissiere, L. et al. Overexpression of human aspartyl(asparaginyl)betahydroxylase in hepatocellular carcinoma and cholangiocarcinoma. J. Clin. Invest. 98, 1313-1323 (1996).

23. Ince, N., de la Monte, S. M. \& Wands, J. R. Overexpression of human aspartyl (asparaginyl) $\beta$-hydroxylase is associated with malignant transformation. Cancer Res. 60, 1261-1266 (2000)

24. Kanwal, M., Smahel, M., Olsen, M., Smahelova, J. \& Tachezy, R. Aspartate $\beta$ hydroxylase as a target for cancer therapy. J. Exp. Clin. Cancer Res. 39, 163 (2020). 
25. Zheng, W., Wang, X., Hu, J., Bai, B. \& Zhu, H. Diverse molecular functions of aspartate $\beta$-hydroxylase in cancer (Review). Oncol. Rep. 44, 2364-2372 (2020).

26. Greve, J. M., Pinkham, A. M. \& Cowan, J. A. Human aspartyl (asparaginyl) hydroxylase. A multifaceted enzyme with broad intra- and extra-cellular activity. Metallomics 13, mfab044 (2021).

27. Brewitz, L., Tumber, A. \& Schofield, C. J. Kinetic parameters of human aspartate/asparagine- $\beta$-hydroxylase suggest that it has a possible function in oxygen sensing. J. Biol. Chem. 295, 7826-7838 (2020).

28. Stenflo, J. et al. Hydroxylation of aspartic acid in domains homologous to the epidermal growth factor precursor is catalyzed by a 2-oxoglutarate-dependent dioxygenase. Proc. Natl. Acad. Sci. USA 86, 444-447 (1989).

29. Gronke, R. S. et al. Aspartyl $\beta$-hydroxylase: in vitro hydroxylation of a synthetic peptide based on the structure of the first growth factor-like domain of human factor IX. Proc. Natl. Acad. Sci. USA 86, 3609-3613 (1989).

30. Korioth, F., Gieffers, C. \& Frey, J. Cloning and characterization of the human gene encoding aspartyl $\beta$-hydroxylase. Gene 150, 395-399 (1994).

31. Pfeffer, I. et al. Aspartate/asparagine- $\beta$-hydroxylase crystal structures reveal an unexpected epidermal growth factor-like domain substrate disulfide pattern. Nat. Commun. 10, 4910 (2019).

32. Kaelin, W. G. \& Ratcliffe, P. J. Oxygen sensing by metazoans: the central role of the HIF hydroxylase pathway. Mol. Cell 30, 393-402 (2008).

33. Schofield, C. J. \& Ratcliffe, P. J. Oxygen sensing by HIF hydroxylases. Nat. Rev. Mol. Cell Biol. 5, 343-354 (2004).

34. Lando, D., Peet, D. J., Whelan, D. A., Gorman, J. J. \& Whitelaw, M. L. Asparagine hydroxylation of the HIF transactivation domain: a hypoxic switch. Science 295, 858-861 (2002).

35. McNeill, L. A. et al. Hypoxia-inducible factor asparaginyl hydroxylase (FIH-1) catalyses hydroxylation at the $\beta$-carbon of asparagine-803. Biochem. J. 367, 571-575 (2002).

36. Mahon, P. C., Hirota, K. \& Semenza, G. L. FIH-1: a novel protein that interacts with HIF- $1 \alpha$ and VHL to mediate repression of HIF-1 transcriptional activity. Genes Dev. 15, 2675-2686 (2001).

37. Koivunen, P., Hirsilä, M., Günzler, V., Kivirikko, K. I. \& Myllyharju, J. Catalytic properties of the asparaginyl hydroxylase (FIH) in the oxygen sensing pathway are distinct from those of its prolyl 4-hydroxylases. J. Biol. Chem. 279, 9899-9904 (2004).

38. Cockman, M. E. et al. Posttranslational hydroxylation of ankyrin repeats in IкB proteins by the hypoxia-inducible factor (HIF) asparaginyl hydroxylase, factor inhibiting HIF (FIH). Proc. Natl Acad. Sci. USA 103, 14767-14772 (2006).

39. Coleman, M. L. et al. Asparaginyl hydroxylation of the notch ankyrin repeat domain by factor inhibiting hypoxia-inducible factor. J. Biol. Chem. 282, 24027-24038 (2007).

40. Ferguson, J. E. et al. ASB4 is a hydroxylation substrate of FIH and promotes vascular differentiation via an oxygen-dependent mechanism. Mol. Cell. Biol. 27, 6407-6419 (2007).

41. Yang, M. et al. Factor-inhibiting hypoxia-inducible factor (FIH) catalyses the post-translational hydroxylation of histidinyl residues within ankyrin repeat domains. FEBS J. 278, 1086-1097 (2011)

42. Yang, M. et al. Substrate selectivity analyses of factor inhibiting hypoxiainducible factor. Angew. Chem. Int. Ed. 52, 1700-1704 (2013).

43. Choi, H. et al. A human protein hydroxylase that accepts D-residues. Commun. Chem. 3, 52 (2020).

44. Holt-Martyn, J. P. et al. Structure-activity relationship and crystallographic studies on 4-hydroxypyrimidine HIF prolyl hydroxylase domain inhibitors. ChemMedChem 15, 270-273 (2020).

45. Chan, M. C. et al. Potent and selective triazole-based inhibitors of the hypoxia-inducible factor prolyl-hydroxylases with activity in the murine brain. PLOS ONE 10, e0132004 (2015).

46. Mosavi, L. K. \& Minor, D. L. \& Peng, Z.-y. Consensus-derived structural determinants of the ankyrin repeat motif. Proc. Natl Acad. Sci. USA 99, 16029-16034 (2002).

47. Kelly, L., McDonough, M. A., Coleman, M. L., Ratcliffe, P. J. \& Schofield, C. J. Asparagine $\beta$-hydroxylation stabilizes the ankyrin repeat domain fold. Mol. BioSyst. 5, 52-58 (2009).

48. Wilkins, A. L. \& Lu, Y. Extractives from New Zealand honeys. 5. Aliphatic dicarboxylic acids in New Zealand rewarewa (Knightea excelsa) honey. J. Agric. Food Chem. 43, 3021-3025 (1995).

49. Mukherjee, D. \& Laloraya, M. M. Metabolism of $\gamma$-methyl- $\alpha$-ketoglutaric acid, $\gamma$-methylene- $\alpha$-ketoglutaric acid and other keto acids during the seedling growth in Tamarindus indica. Biochem. Physiol. Pflanz. 166, 429-436 (1974).

50. Virtanen, A. I. \& Berg, A.-M. New aminodicarboxylic acids and corresponding a-keto acids in phyllitis scolopendrium. Acta Chem. Scand. 9, 553-554 (1955).

51. Díaz, R. et al. Told through the wine: a liquid chromatography-mass spectrometry interplatform comparison reveals the influence of the global approach on the final annotated metabolites in non-targeted metabolomics. J. Chromatogr. A 1433, 90-97 (2016).
52. McDonough, M. A. et al. Selective inhibition of factor inhibiting hypoxiainducible factor. J. Am. Chem. Soc. 127, 7680-7681 (2005).

53. Tarhonskaya, H. et al. Kinetic investigations of the role of factor inhibiting hypoxia-inducible factor (FIH) as an oxygen sensor. J. Biol. Chem. 290, 19726-19742 (2015)

54. Hutchinson, S. E. et al. Enabling lead discovery for histone lysine demethylases by high-throughput RapidFire mass spectrometry. J. Biomol. Screen. 17, 39-48 (2011).

55. Nowak, R. P. et al. First-in-class inhibitors of the ribosomal oxygenase MINA53. manuscript under revision.

56. Ingraham, L. et al. A plasma concentration of $\alpha$-ketoglutarate influences the kinetic interaction of ligands with OAT1. Mol. Pharmacol. 86, 86-95 (2014).

57. Siess, E. A., Brocks, D. G., Lattke, H. K. \& Wieland, O. H. Effect of glucagon on metabolite compartmentation in isolated rat liver cells during gluconeogenesis from lactate. Biochem. J. 166, 225-235 (1977).

58. Thirstrup, K. et al. Endogenous 2-oxoglutarate levels impact potencies of competitive HIF prolyl hydroxylase inhibitors. Pharmacol. Res. 64, 268-273 (2011).

59. Brewitz, L., Tumber, A., Pfeffer, I., McDonough, M. A. \& Schofield, C. J. Aspartate/asparagine- $\beta$-hydroxylase: a high-throughput mass spectrometric assay for discovery of small molecule inhibitors. Sci. Rep. 10, 8650 (2020).

60. Lancaster, D. E. et al. Disruption of dimerization and substrate phosphorylation inhibit factor inhibiting hypoxia-inducible factor (FIH) activity. Biochem. J. 383, 429-437 (2004).

61. Elkins, J. M. et al. Structure of factor-inhibiting hypoxia-inducible factor (HIF) reveals mechanism of oxidative modification of HIF-1a. J. Biol. Chem. 278, 1802-1806 (2003).

62. Cockman, M. E., Webb, J. D., Kramer, H. B., Kessler, B. M. \& Ratcliffe, P. J. Proteomics-based identification of novel factor inhibiting hypoxia-inducible factor (FIH) substrates indicates widespread asparaginyl hydroxylation of ankyrin repeat domain-containing proteins. Mol. Cell. Proteom. 8, 535-546 (2009).

63. Cornish-Bowden, A. A simple graphical method for determining the inhibition constants of mixed, uncompetitive and non-competitive inhibitors. Biochem. J. 137, 143-144 (1974).

64. Losman, J.-A. \& Kaelin, W. G. What a difference a hydroxyl makes: mutant IDH, (R)-2-hydroxyglutarate, and cancer. Genes Dev. 27, 836-852 (2013).

65. Dang, L. \& Su, S.-S. M. Isocitrate dehydrogenase mutation and (R)-2hydroxyglutarate: from basic discovery to therapeutics development. Annu. Rev. Biochem. 86, 305-331 (2017)

66. Takeuchi, H. et al. Two novel protein $O$-glucosyltransferases that modify sites distinct from POGLUT1 and affect Notch trafficking and signaling. Proc. Natl Acad. Sci. USA 115, E8395-E8402 (2018).

67. Metzen, E. et al. Intracellular localisation of human HIF-1a hydroxylases: implications for oxygen sensing. J. Cell Sci. 116, 1319-1326 (2003).

68. Jia, S. et al. cDNA cloning and expression of bovine aspartyl (asparaginyl) $\beta$ hydroxylase. J. Biol. Chem. 267, 14322-14327 (1992).

69. Aik, W., McDonough, M. A., Thalhammer, A., Chowdhury, R. \& Schofield, C. $\mathrm{J}$. Role of the jelly-roll fold in substrate binding by 2-oxoglutarate oxygenases. Curr. Opin. Struct. Biol. 22, 691-700 (2012).

70. Hamada, S. et al. Synthesis and activity of $\mathrm{N}$-oxalylglycine and its derivatives as Jumonji C-domain-containing histone lysine demethylase inhibitors. Bioorg. Med. Chem. Lett. 19, 2852-2855 (2009).

71. Rose, N. R. et al. Inhibitor scaffolds for 2-oxoglutarate-dependent histone lysine demethylases. J. Med. Chem. 51, 7053-7056 (2008).

72. Rose, N. R. et al. Selective inhibitors of the JMJD2 histone demethylases: combined nondenaturing mass spectrometric screening and crystallographic approaches. J. Med. Chem. 53, 1810-1818 (2010).

73. Sudhamalla, B. et al. Complementary steric engineering at the protein-ligand interface for analogue-sensitive TET oxygenases. J. Am. Chem. Soc. 140, 10263-10269 (2018)

74. Hélaine, V., Rossi, J., Gefflaut, T., Alaux, S. \& Bolte, J. Synthesis of 4,4disubstituted L-glutamic acids by enzymatic transamination. Adv. Synth. Catal. 343, 692-697 (2001).

75. Epstein, A. C. R. et al. C. elegans EGL-9 and mammalian homologs define a family of dioxygenases that regulate HIF by prolyl hydroxylation. Cell 107, 43-54 (2001)

76. Wu, Y., Li, Z., McDonough, M. A., Schofield, C. J. \& Zhang, X. Inhibition of the oxygen-sensing asparaginyl hydroxylase factor inhibiting hypoxiainducible factor: a potential hypoxia response modulating strategy. J. Med. Chem. 64, 7189-7209 (2021).

77. Aihara, A. et al. A cell-surface $\beta$-hydroxylase is a biomarker and therapeutic target for hepatocellular carcinoma. Hepatology 60, 1302-1313 (2014).

78. Dong, X. et al. Aspartate $\beta$-hydroxylase expression promotes a malignant pancreatic cellular phenotype. Oncotarget 6, 1231-1248 (2014).

79. Nagaoka, K. et al. Targeting aspartate beta-hydroxylase with the small molecule inhibitor MO-I-1182 suppresses cholangiocarcinoma metastasis. Dig. Dis. Sci. 66, 1080-1089 (2021). 
80. Brewitz, L. et al. Synthesis of novel pyridine-carboxylates as small-molecule inhibitors of human aspartate/asparagine- $\beta$-hydroxylase. ChemMedChem 15, 1139-1149 (2020).

81. Brewitz, L., Nakashima, Y., Tumber, A., Salah, E. \& Schofield, C. J. Fluorinated derivatives of pyridine-2,4-dicarboxylate are potent inhibitors of human 2-oxoglutarate dependent oxygenases. J. Fluor. Chem. 247, 109804 (2021).

82. Hewitson, K. S. et al. Hypoxia-inducible Factor (HIF) asparagine hydroxylase is identical to Factor Inhibiting HIF (FIH) and is related to the cupin structural family. J. Biol. Chem. 277, 26351-26355 (2002).

83. Zhang, J.-H., Chung, T. D. Y. \& Oldenburg, K. R. A simple statistical parameter for use in evaluation and validation of high throughput screening assays. J. Biomol. Screen. 4, 67-73 (1999).

84. Vonrhein, C. et al. Data processing and analysis with the autoPROC toolbox. Acta Cryst. D. 67, 293-302 (2011).

85. Tickle, I. J. et al., STARANISO (http://staraniso.globalphasing.org/cgi-bin/ staraniso.cgi). Cambridge, United Kingdom: Global Phasing Ltd. (2018).

86. Winter, G. xia2: an expert system for macromolecular crystallography data reduction. J. Appl. Cryst. 43, 186-190 (2010).

87. McCoy, A. J. et al. Phaser crystallographic software. J. Appl. Cryst. 40, 658-674 (2007).

88. Adams, P. D. et al. PHENIX: a comprehensive python-based system for macromolecular structure solution. Acta Cryst. D. 66, 213-221 (2010).

89. Emsley, P., Lohkamp, B., Scott, W. G. \& Cowtan, K. Features and development of Coot. Acta Cryst. D. 66, 486-501 (2010).

90. Afonine, P. V. et al. Towards automated crystallographic structure refinement with phenix.refine. Acta Cryst. D. 68, 352-367 (2012).

91. DeLano, W. L. The PyMOL molecular graphics system. (De Lano Scientific, 2002).

92. Liebschner, D. et al. Polder maps: improving OMIT maps by excluding bulk solvent. Acta Cryst. D. 73, 148-157 (2017).

93. Hill, A. V. The possible effects of the aggregation of the molecules of haemoglobin on its dissociation curves. J. Physiol. 40, iv-vii (1910).

\section{Acknowledgements}

This research was funded in part by the Wellcome Trust (106244/Z/14/Z). For the purpose of open access, the author has applied a CC BY public copyright license to any Author Accepted Manuscript version arising from this submission. We thank Cancer Research UK (C8717/A18245) and the Biotechnology and Biological Sciences Research Council (BB/J003018/1 and BB/R000344/1) for funding. Y.N. thanks JSPS for an Overseas Research Fellowship (2020060219) and the Daiichi Sankyo Foundation of Life Science. L.B. thanks the Deutsche Forschungsgemeinschaft for a fellowship (BR 5486/2-1).
We thank the Diamond Light Source and staff for the allocation of beam time and support.

\section{Author contributions}

Y.N. performed crystallizations and solved and refined the FIH crystal structures. L.B. and A.T. performed assays. L.B. synthesized 2OG derivatives. E.S. and L.B. produced and purified enzymes. All authors analyzed data. L.B., Y.N., and C.J.S. wrote the manuscript.

\section{Competing interests}

The authors declare no competing interests.

\section{Additional information}

Supplementary information The online version contains supplementary material available at https://doi.org/10.1038/s41467-021-26673-2.

Correspondence and requests for materials should be addressed to Christopher J. Schofield.

Peer review information Nature Communications thanks the anonymous reviewers for their contribution to the peer review of this work

Reprints and permission information is available at http://www.nature.com/reprints

Publisher's note Springer Nature remains neutral with regard to jurisdictional claims in published maps and institutional affiliations.

\section{(c) (i)}

Open Access This article is licensed under a Creative Commons Attribution 4.0 International License, which permits use, sharing, adaptation, distribution and reproduction in any medium or format, as long as you give appropriate credit to the original author(s) and the source, provide a link to the Creative Commons license, and indicate if changes were made. The images or other third party material in this article are included in the article's Creative Commons license, unless indicated otherwise in a credit line to the material. If material is not included in the article's Creative Commons license and your intended use is not permitted by statutory regulation or exceeds the permitted use, you will need to obtain permission directly from the copyright holder. To view a copy of this license, visit http://creativecommons.org/ licenses/by/4.0/

(C) The Author(s) 2021 